\title{
GAUSS - genesis of asteroids and evolution of the solar system
}

\section{A sample return mission to Ceres}

Xian Shi ${ }^{1,2}$ (D) . Julie Castillo-Rogez ${ }^{3}$ Henry Hsieh ${ }^{4} \cdot \mathrm{Hejiu} \mathrm{Hui}^{5}$. Wing-Huen $\mathrm{Ip}^{6} \cdot$ Hanlun Lei ${ }^{7}$. Jian-Yang $\mathrm{Li}^{4} \cdot$ Federico Tosi ${ }^{8} \cdot$ Liyong Zhou $^{7}$. Jessica Agarwal ${ }^{1,9}$. Antonella Barucci ${ }^{10}$. Pierre Beck ${ }^{11}$. Adriano Campo Bagatin ${ }^{12}$. Fabrizio Capaccioni ${ }^{8}$. Andrew J. Coates ${ }^{13}$. Gabriele Cremonese $^{14}$. Rene Duffard ${ }^{15}$. Manuel Grande ${ }^{16}$. Ralf Jaumann ${ }^{17}$. Geraint H. Jones ${ }^{13}$. Esa Kallio ${ }^{18}$. Yangting Lin ${ }^{19}$. Olivier Mousis ${ }^{20}$. Andreas Nathues $^{1}$. Jürgen Oberst ${ }^{21} \cdot$ Holger Sierks $^{1}$. Stephan Ulamec ${ }^{22}$. Mingyuan Wang ${ }^{23} \cdot$ The GAUSS Team

Received: 30 July 2020 / Accepted: 23 September 2021 / Published online: 15 October 2021

(C) The Author(s) 2021

\section{Abstract}

The goal of Project GAUSS (Genesis of Asteroids and evolUtion of the Solar System) is to return samples from the dwarf planet Ceres. Ceres is the most accessible candidate of ocean worlds and the largest reservoir of water in the inner Solar System. It shows active volcanism and hydrothermal activities in recent history. Recent evidence for the existence of a subsurface ocean on Ceres and the complex geochemistry suggest past habitability and even the potential for ongoing habitability. GAUSS will return samples from Ceres with the aim of answering the following top-level scientific questions:

- What is the origin of Ceres and what does this imply for the origin of water and other volatiles in the inner Solar System?

- What are the physical properties and internal structure of Ceres? What do they tell us about the evolutionary and aqueous alteration history of dwarf planets?

- What are the astrobiological implications of Ceres? Is it still habitable today?

- What are the mineralogical connections between Ceres and our current collections of carbonaceous meteorites?

Keywords Ceres - Dwarf planet - Sample return • Ocean world · Habitability · Voyage 2050

Xian Shi

shi@shao.ac.cn

Extended author information available on the last page of the article. 


\section{Background}

Though the Rosetta mission to comet 67P/Churyumov-Gerasimenko came to an end only recently, in 2016, it is important to recall that the planning activity eventually leading to its approval by ESA was initiated more than three decades earlier in 1983. An equally, if not more, ambitious project in the framework of "Voyage 2050" is proposed here. The target is the innermost dwarf planet, Ceres, which was discovered on New Year's Day of 1801, by the Italian astronomer Giuseppe Piazzi at Palermo Observatory. At the time of its discovery, Ceres was considered to be the missing planet between the orbits of Mars and Jupiter as predicted by the Titius-Bode law [38]. Ceres' location was confirmed in December the same year using the orbital elements calculated by then 24-year-old Carl Friedrich Gauss [111]. The name of the project with the acronym of GAUSS for "Genesis of Asteroids and evolUtion of the Solar System" is partly a tribute to this scientific episode of great importance in astronomy and planetary science.

\section{Scientific rationale}

\subsection{Ceres in the history of the solar system}

The formation of the oldest solids found in the Solar System - calcium-aluminium inclusions (CAIs) - dates back 4567.5 Myr [22]. After that, the formation of gas giants must finish before the dispersal of the protoplanetary gas disc, which lasts 2$10 \mathrm{Myr}[4,46]$. Then, about 30-100 Myr later, the terrestrial planets formed in the planetesimal disc [50].

The planetesimal disc not only provided all the materials that constitute the terrestrial planets and cores of gas giants, but also exerted perturbing forces on the grown planets $[40,55]$. This perturbation might have continuously modified the planetary orbits in a relatively gentle way [32]. Before the dispersal of the gas disc, planets could have already experienced migration due to the interaction with the massive gas disc [118]. During the planetary migration afterwards, mechanisms such as low-order mean motion resonance between planets brought up abrupt variations in the orbital configuration of planets [115]. The interaction between the planetesimal disc and migrating planets/protoplanets reconfigured the structure of the Solar System [36, $70,118]$. However, the growth and evolution of terrestrial planets and asteroids (in a wide radial range from Mars' orbit to the Kuiper belt) in the early stages of the Solar System are not fully understood.

Given that their properties depend on their formation circumstances and evolutionary processes experienced since their formation, small bodies (e.g., asteroids, comets, and trans-Neptunian objects) in the Solar System are of interest to researchers because they provide a way to probe the protoplanetary disc from which our Solar System formed $[6,52,103]$. By determining the chemical and physical properties of various small bodies in our Solar System, we can gain insight into chemical and thermal conditions in different areas of the protoplanetary disc and also investigate the chemical, thermal, collisional, and dynamical processes that have shaped those 


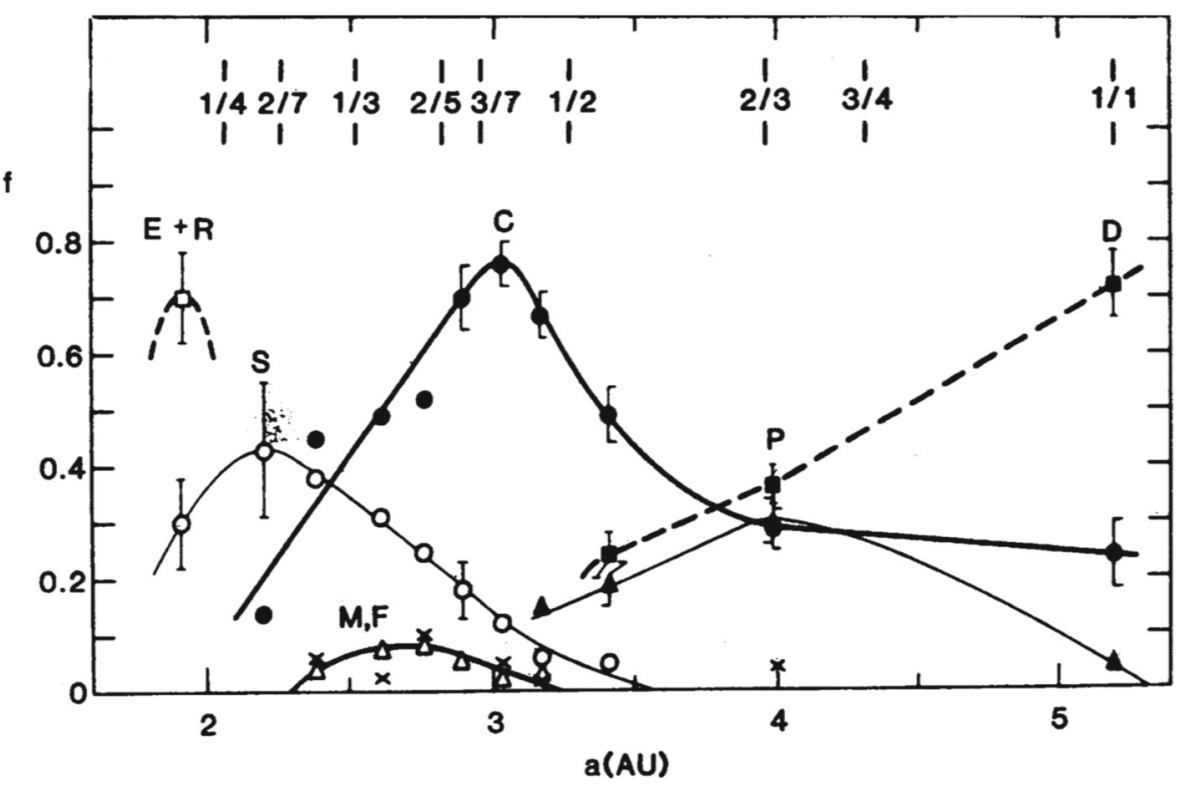

Fig. 1 Relative distribution of taxonomic types for a bias-corrected sample of 656 main-belt asteroids. Letters indicate different compositional types. Refer to Table 1 in [37] for the definition of each type. Locations of major resonances with Jupiter are indicated at the top. (Figure reprinted from [37])

populations since their formation. A successful scenario of planet migration should provide the mechanisms that were able to efficiently deliver planetesimals from different zones to the main belt. In addition, the structure of the main belt, the mass depletion, the proper excitation of orbital eccentricities and inclinations, should all be reproduced.

One of the earliest attempts to map the compositional structure of our asteroid belt and use it to infer information about the origin and evolution of the Solar System was performed by [37]. They found a systematic distribution of compositional types of asteroids in the main asteroid belt that they suggested was consistent with chemical condensation models of the Solar System (Fig. 1). The authors concluded that it was unlikely that this distribution could be explained by the chaotic transport of objects from different regions of the Solar System into the asteroid belt, and proposed instead that it indicated that the asteroids formed at or near their present locations.

The work of [37] has since undergone a major update in the form of a study by [27], who used the Sloan Digital Sky Survey (SDSS) Moving Object Catalog data to derive taxonomic classifications for about 35000 objects, including objects as small as $5 \mathrm{~km}$ in diameter. The inclusion of much smaller objects in this sample than were previously available, as well as the computation of compositional distributions by mass (instead of by number, see Fig. 2 in [28]) suggest that the asteroid belt preserves a history of Solar System evolution that is far more complex than previously thought $[28,36,63,118]$, with asteroids of various taxonomic types scattered throughout the 
asteroid belt, including in regions where they are not expected based on dynamically static Solar System formation models. Instead, the history of the Solar System as recorded by main-belt asteroids likely includes relatively brief periods of dramatic mixing caused by giant planetary migration, e.g., those proposed as parts of the well-known Nice and Grand Tack models [63, 64, 70, 115, 115, 118] as well as less dramatic but ongoing processes such as collisions and small body migration due to mean-motion resonances with the giant planets and the Yarkovsky effect (e.g., [7, $8,31,35,88])$.

The fraction and composition of ice in a body is of particular interest due to the strong temperature constraints they provide. The so-called "snow line" refers to the distance from the Sun at which the temperature dips below the condensation temperature of water, causing it to freeze into solid ice and then become incorporated into accreting planetesimals. The exact location of the snow line in our protoplanetary disc was dependent on a variety of poorly constrained environmental conditions including opacity, mass density, and accretion rate in the disc, and is also thought to have shifted with time as planetesimal accretion progressed and the aforementioned properties of the disc changed [71].

Bodies formed in the outer Solar System such as between Jupiter and Neptune (the original accretion zone of current Oort Cloud objects; [40, 120]) and beyond the orbit of Neptune (the Kuiper Belt) are well beyond the snow line. Closer to the Sun, the situation is less certain. Observations of asteroids suggest that the snow line probably existed as close as 2.5 au from the Sun [37, 52], but theoretical studies [19, $62,97]$ have placed it as close in as the orbit of Mars, or even closer. If this is true, it means that objects throughout the main asteroid belt could have incorporated some water ice at the time of their formation.

Evidence of past and present-day ice has in fact been found in main-belt asteroids. Studies of meteorites linked to the asteroid belt as well as remote spectroscopic observations of asteroids have revealed the presence of aqueously altered minerals, indications that liquid water was once present $[47,53,90]$. Meanwhile, spectroscopic evidence of water ice frost has been reported for various main-belt asteroids including (24) Themis [41, 89, 110], while some main-belt objects have even been observed to exhibit comet-like activity that has been attributed to the sublimation of present-day volatile ices, i.e., the so-called main-belt comets [48]. The location, abundance, distribution, and inferred water content of currently and formerly icy main-belt objects provide valuable clues for discerning the primordial location and evolution of the snow line, although this of course is also complicated by the aforementioned likely transport of at least some small bodies from their original formation locations due to giant planet migration and other dynamical processes.

The bulk-rock isotope anomalies of meteorites reveal an isotopic dichotomy: two distinct trends defined by the non-carbonaceous (NC) bodies and the carbonaceous (CC) bodies respectively (Fig. 2). The CC bodies include carbonaceous chondrites, a few ungrouped achondrites, and IIC, IID, IIF, IIIF, IVB iron meteorites. The NC bodies include Earth, the Moon, Mars, ordinary chondrites, enstatite chondrites, and most of achondrites. This dichotomy has been further observed in $\mathrm{Mo}, \mathrm{W}, \mathrm{Ru}$, and $\mathrm{Ni}$ isotopic systematics $[11,58,72,80]$. However, the origins of the isotopic anomalies and their correlations are unknown, although ${ }^{54} \mathrm{Cr}$-enrichment can be attributed to 

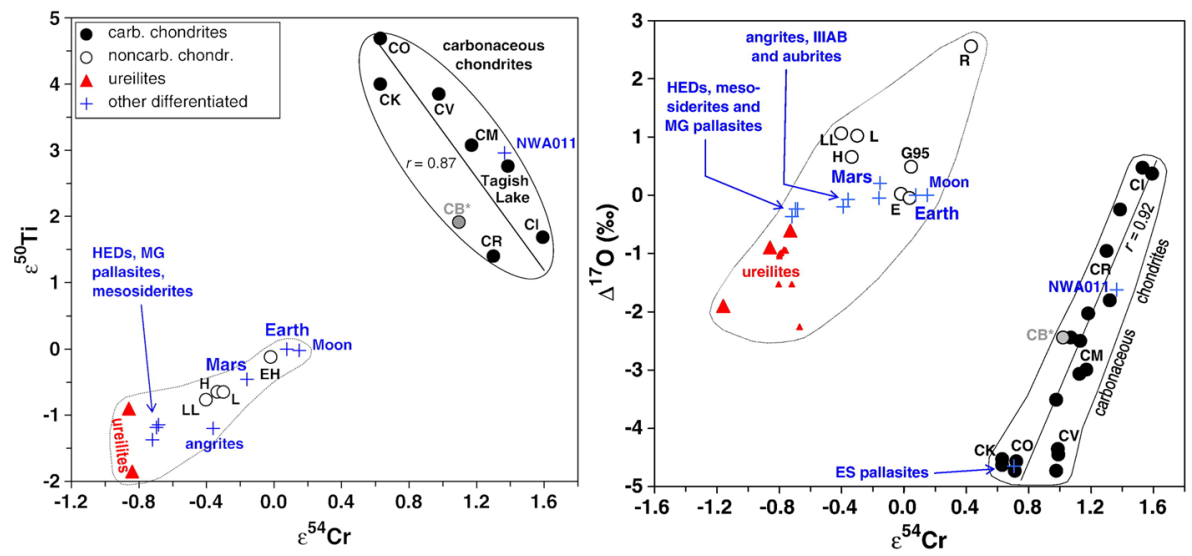

Fig. 2 Dichotomy of stable isotopes among planetary materials. (Figure reprinted from [119])

nano-sized Cr-oxide probably ejected from supernovas [83]. Based on the isotopic dichotomy, [119] proposed that the CC bodies formed in the outer Solar System and the NC bodies formed in the inner Solar System. The formation of Jupiter blocked the material exchange between the two groups. As a consequence of radial migration and mass growth of the giant planets in the Solar System, some CC planetesimals and embryos moved inwards from the outer Solar System to the main belt $[58,88$, 118, 119].

Ceres, the largest object in the main belt, preserves many clues to the formation and evolution of the main belt, as well as of the Solar System. The surface material of Ceres is similar to carbonaceous chondrite, though no meteorite has so far been linked with Ceres [91]. Interpretation of the data from the Dawn mission with geochemical simulations show that the surface mineralogy of Ceres is consistent with the aqueous alteration of CI and CM chondrite [15].

The ammonia detected on Ceres [23] suggests that Ceres could have originated in the outer Solar System, and migrated to its current neighbourhood. However, this scenario still misses definitive evidence. Ceres could have formed in the outer Solar System or formed in situ and accreted materials radially transported from the outer Solar System [68]. The stable isotope anomalies of samples from Ceres could provide ground truth on its formation location (Fig. 2). Furthermore, geochemical information in returned samples could be used to understand the evolution path of Ceres.

\subsection{Results from the dawn mission}

The Dawn mission performed a 3.5 years rendezvous with Ceres starting from early 2015, mapping its geomorphology at resolutions down to $\sim 35 \mathrm{~m}$ globally [92], derived surface mineralogy at resolutions down to $140 \mathrm{~m}[33]$, the top layer $(\sim 0.5-$ $1 \mathrm{~m}$ ) elemental abundance at effective resolutions of tens of $\mathrm{km}$, as well as the gravity field to 18 degrees of spherical harmonics[56]. During the last mission phase, Dawn 
entered a long elliptical orbit with an altitude of $35 \mathrm{~km}$ at periapsis for a detailed study of the narrow longitudinal strip at about $240^{\circ} \mathrm{E}$ that stretches from north of Occator crater and goes up to Azacca crater on the other hemisphere. During this phase, images were acquired with resolutions as high as $3.5 \mathrm{~m} /$ pixel.

The Dawn results confirmed that Ceres is volatile-rich, has a partially differentiated interior, and has experienced global aqueous alteration. Dawn revealed Ceres as a geologically active dwarf planet with brine-driven volcanism as recently as of a few Myr and probably even at present. Ammoniated phyllosilicates are distributed all over the surface of Ceres [3, 23]. The incorporation of ammonia in Ceres' surface mineralogy is an indication that Ceres may have accreted materials from the giant planet formation region.

The surface layer of Ceres is rich in water of hydration and water ice. The abundance of water ice is relatively low towards the equator and high towards the polar regions [81]. The latitudinal variation is consistent with the evolution of subsurface water ice as controlled by the thermal condition on the surface and shallow subsurface of Ceres [99, 100] and the low obliquity of Ceres [96]. Water ice has been directly identified in about ten specific places on the surface, near rim shadows in fresh craters at latitudes poleward of $30^{\circ}[20,21]$, and inside the permanently shadowed craters in the polar regions $[79,101]$. The existence of water ice in the first few $\mathrm{km}$ of the crust is also inferred by analyzing lobate morphologies [98].

The abundant water ice on Ceres (Fig. 3) led to differentiation and aqueous alteration that shaped the mineralogical composition of Ceres in its crust and mantle [15]. Near-infrared data returned by the Visible and InfraRed mapping spectrometer (VIR) on board the Dawn spacecraft detected carbonates and ammonium salts that are previously found only on Earth and Enceladus [24]. The existence of brines at depth played a key role in driving the geological process on Ceres, including volcanism and activities in its geologically recent history [94, 95, 105].

The most prominent geomorphological feature that is considered of volcanic origin is Ahuna Mons (Fig. 4) [94, 95]. Its distinct size, shape, and morphology are consistent with being a volcanic dome formed by extrusions of highly viscous meltbearing material. At the summit is the buildup of a brittle carapace, which partially fractured and disintegrated to generate the slope debris. The bright streaks in the slope debris are rich in Na-carbonates [12, 123, 124]. The gravitational relaxation of the enclosed ductile core shapes the overall topographic profile of the summit, requiring an extruded material of high viscosity. The age of the most recent activity on Ahuna Mons is about $210 \pm 30 \mathrm{Myr}$ [94]. Other possible features involving the presence of melt include smaller domes [108, 109], fractures in the crater floor [9], post-impact modification by the deposition of extended plains material with pits and widely dispersed deposits that form a diffuse veneer on the preexisting surface [57].

Shallow subsurface volatiles are also evident from the many other geomorphological features, such as the pitted terrain [107]. On the other hand, the crater morphology and the simple-to-complex crater transition indicate that Ceres' outer shell is likely neither pure ice nor pure rock, but a mixture of ice, rock, salts and/or clathrates that allows for limited and spatially variable viscous relaxation [5, 45].

The distinctive bright regions within Occator crater are one of the most remarkable features on Ceres observed by Dawn (Fig. 5). Occator crater is about $90 \mathrm{~km}$ in 


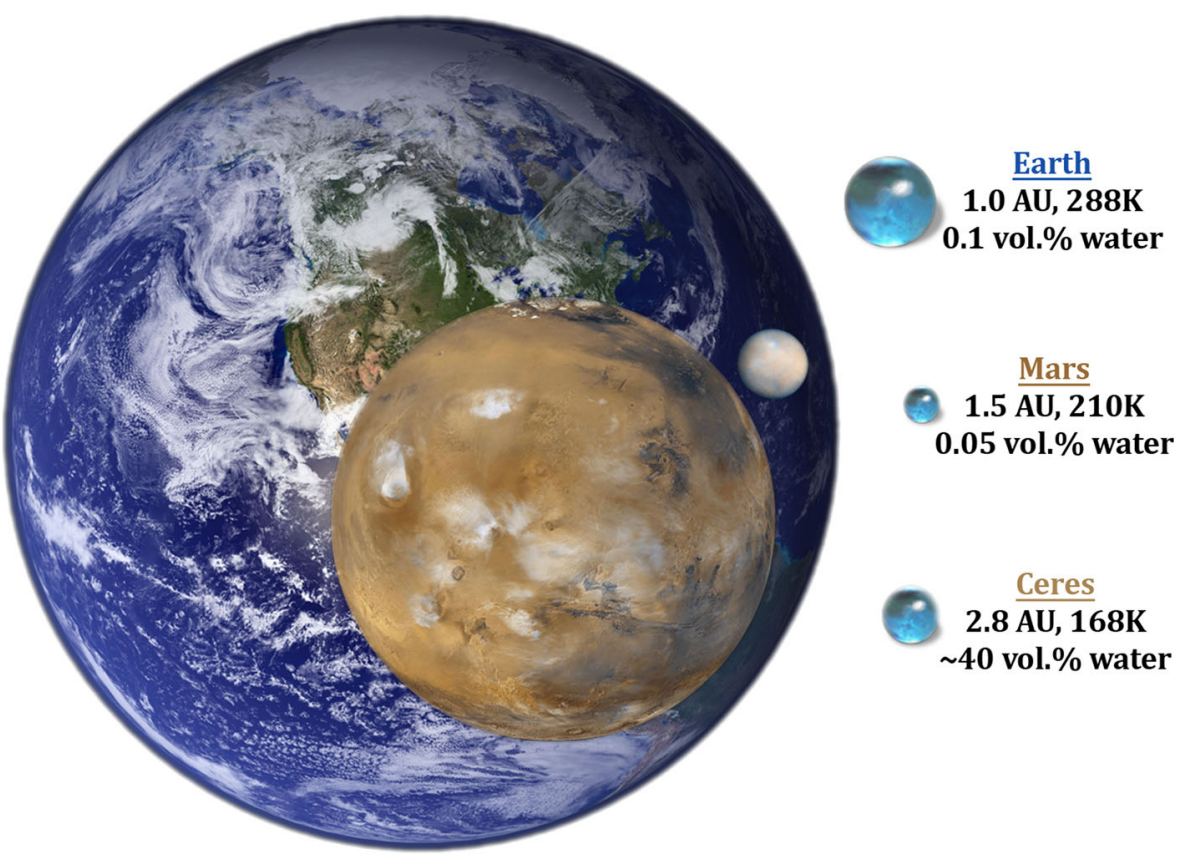

Fig. 3 Comparison view of water abundances on Earth, Mars, and Ceres

diameter, hosting the bright deposits covering the pit-dome complex named Cerealia Facula in the center, and a group of secondary bright deposits named Vinalia Faculae on the east side of the crater floor. While Ceres' average surface contains $\mathrm{Mg}$ - and Ca-carbonates and $\mathrm{Mg}$ - and $\mathrm{NH}_{4}$-phyllosilicates, Occator's faculae contain Na-carbonate, Al-phyllosilicates, and $\mathrm{NH}_{4}$-chloride [86]. Theoretical modelling suggested the possibility of a brine reservoir beneath Occator crater, and the gradual

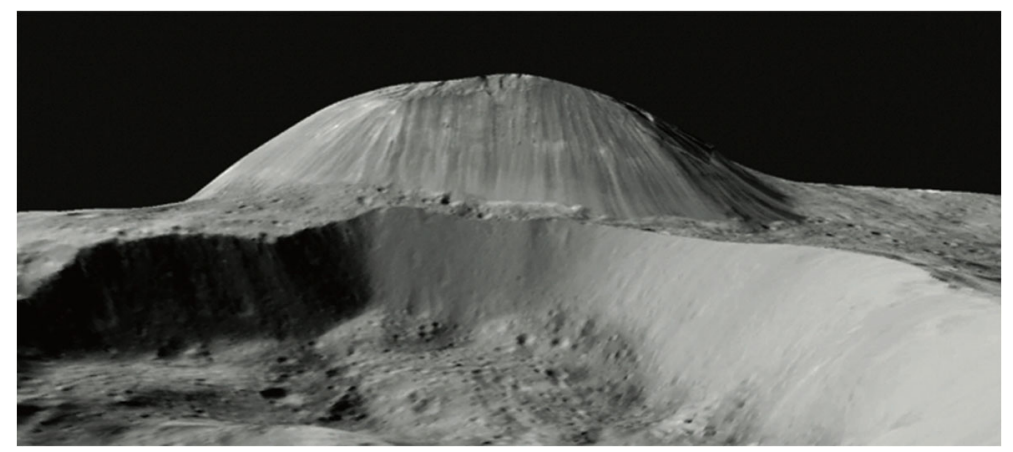

Fig. 4 Perspective view of Ahuna Mons on Ceres from Dawn Framing Camera data (no vertical exaggeration). The mountain is $4 \mathrm{~km}$ high and $17 \mathrm{~km}$ wide in this south-looking view (image credit: NASA/JPL/Caltech/IAPS/MPS/DLR/INAF/ASI) 


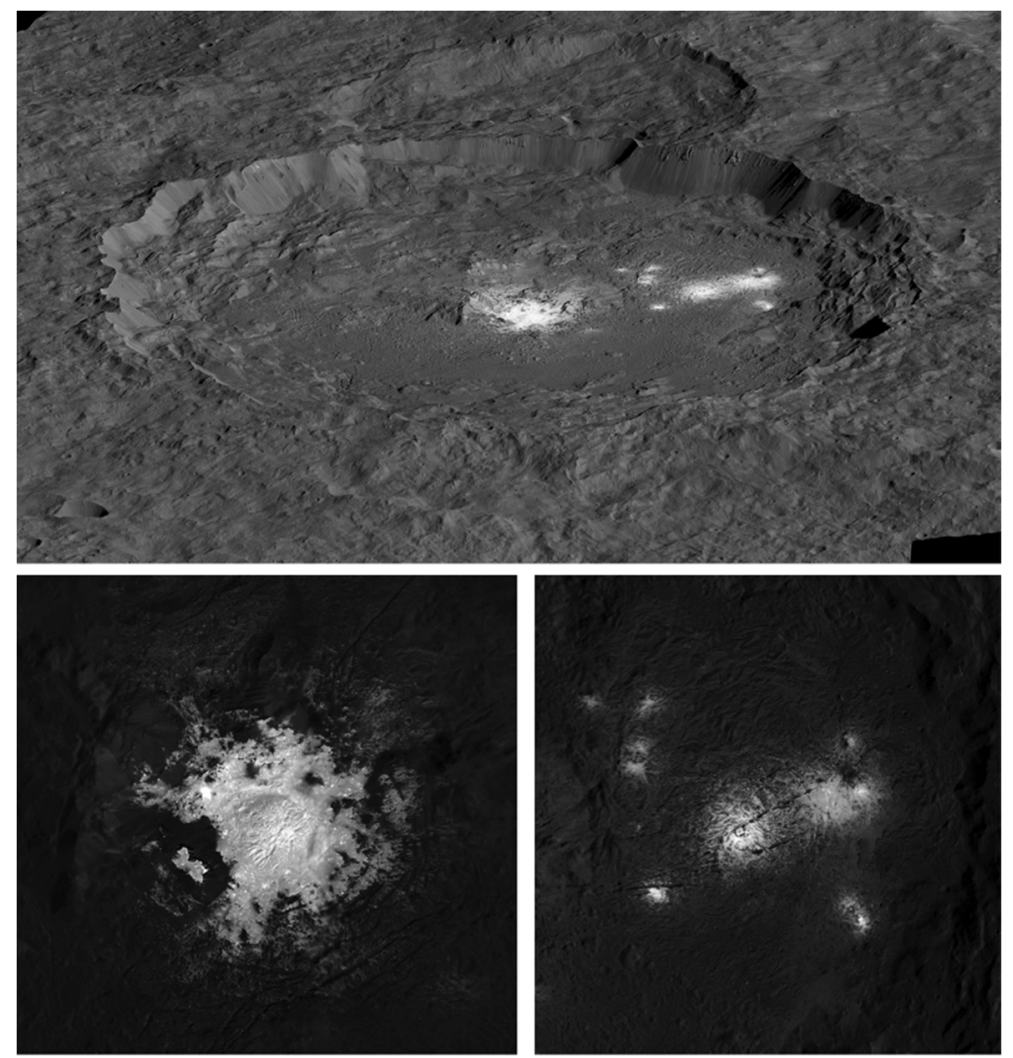

Fig. 5 Top: Perspective view of Occator crater from the south and the bright deposits from Dawn Framing Camera data. The Cerealia Facula is saturated in this brightness stretch. Bottom left: Zoom-in view of the Cerealia Facula at the center of Occator crater shows the details of the bright deposit. Bottom right: Zoom-in view of the Vinalia Faculae (image credit: NASA/JPL/Caltech/IAPS/MPS/DLR/INAF/ASI)

freezing of this reservoir was the driver of briny lavas [84]. The salts lower the eutectic point and extend the duration of volcanic activity on Ceres. Laboratory studies suggest that a slow freezing process $(<30 \mathrm{~K} / \mathrm{min})$ of the exposed ammonium-sodiumcarbonate-chloride-rich brines is most compatible with the observed composition of brines observed in Occator crater [112]. A variety of morphological features are observed in the crater. Linear and concentric fractures on the crater floor are associated with cryomagmatic intrusion, and degassing or desiccation processes for the volatile rich Occator ejecta [10]. The cross-cutting relationship between stratigraphic units indicate that the Cerealia Facula was emplaced in multiple episodes [106]. Crater counting in the Occator ejecta yields an age of about $20 \mathrm{Myr}$ [75], while the age of faculae is only a few Myr [73, 74].

The presence of water ice on the surface of Ceres, as well as the widespread distribution of shallow subsurface water has been associated with the active outgassing of Ceres previously observed from Earth orbit or the Earth-Sun L2 Lagrange point $[1,59]$. However, the observed surface water ice exposures on Ceres do not appear 
to be sufficient to supply the observed water production rate $[60,61,102]$. Multiple attempts to detect water outgassing around Ceres failed, suggesting that water outgassing from Ceres is variable but also does not appear to depend on heliocentric distance [93]. Variation in the amount of water ice was detected on the wall of the Juling crater, indicating the possible existence of a seasonal water cycle [85]. Another hypothesis proposes that the solar energetic particles might be a driver of outgassing [116]. The water loss mechanisms on Ceres and the characteristics of its transient water exosphere are therefore still uncertain.

Gravitational data and geophysical modelling suggested that Ceres is partially differentiated to a $\sim 40 \mathrm{~km}$ thick and strong crust composed of rock, ice, salts, and/or clathrates with no more than $30 \%$ water ice [5, 30, 34]. Below the crust is a denser rocky mantle with a relatively weak upper layer potentially with brine-filled pore space that controls the global shape of Ceres [34]. The possibility of a dehydrated rocky core below $100 \mathrm{~km}$ cannot be ruled out [54].

VIR first detected an organic absorption feature at 3.4- $\mu \mathrm{m}$ on Ceres. This signature is diagnostic of organic matter and is mainly localized on a broad region of $\sim 1000 \mathrm{~km}^{2}$ close to the $\sim 50 \mathrm{~km}$ Ernutet crater [25]. The shape of the $3.4-\mu \mathrm{m}$ band and the lack of an associated 3.25- $\mu \mathrm{m}$ feature could exclude organics with a high content of aromatic carbon such as anthraxolites as main carriers of the features on Ceres, in favour of hydrocarbons rich in aliphatic carbon [25]. Laboratory studies have found organic-rich analogues that could reproduce absorption bands in the VIR spectra [117]. However, the exact nature and concentraton history of the organic matter is still unclear, making this a compelling scientific question for any future space mission to Ceres [117]. Furthermore, organic compounds possibly also exist on top of Cerealia Facula, the brightest spot located roughly in the middle of crater Occator [26].

The volcanism and hydrothermal activity in the recent history of Ceres, the existence of brines on a global scale at the present suggest an active planetary body that could have strong astrobiological significance. The occurrence on Ceres of ammoniabearing hydrated minerals, water ice, carbonates, salts, and organic material, revealed a complex chemical environment, potentially favourable to prebiotic chemistry in a subsurface aqueous environment [25]. The ability to detect, determine, and quantify any organics on Ceres is a clear step toward assessing habitability [16].

Dawn findings have placed Ceres among Solar System "ocean worlds", bodies that host current liquid ocean (not necessarily global, according to [44]). Originally classified as a "candidate" ocean world in NASA's Roadmap to Ocean Worlds [44], Ceres is now suggested as a "real" ocean world in the light of latest results from analyses of Dawn data acquired during its extended mission ([13] and references therein), noting however that the occurrence of liquid may be limited to local or regional reservoirs. In summary, as illustrated in Fig. 6, Dawn revealed the great scientific significance of Ceres:

- Rich in water ice and other volatiles relevant to our understanding of the history of volatiles and organic matter in the inner Solar System

- Formation and evolutionary history representative of ice-rich objects in the outer Solar System 


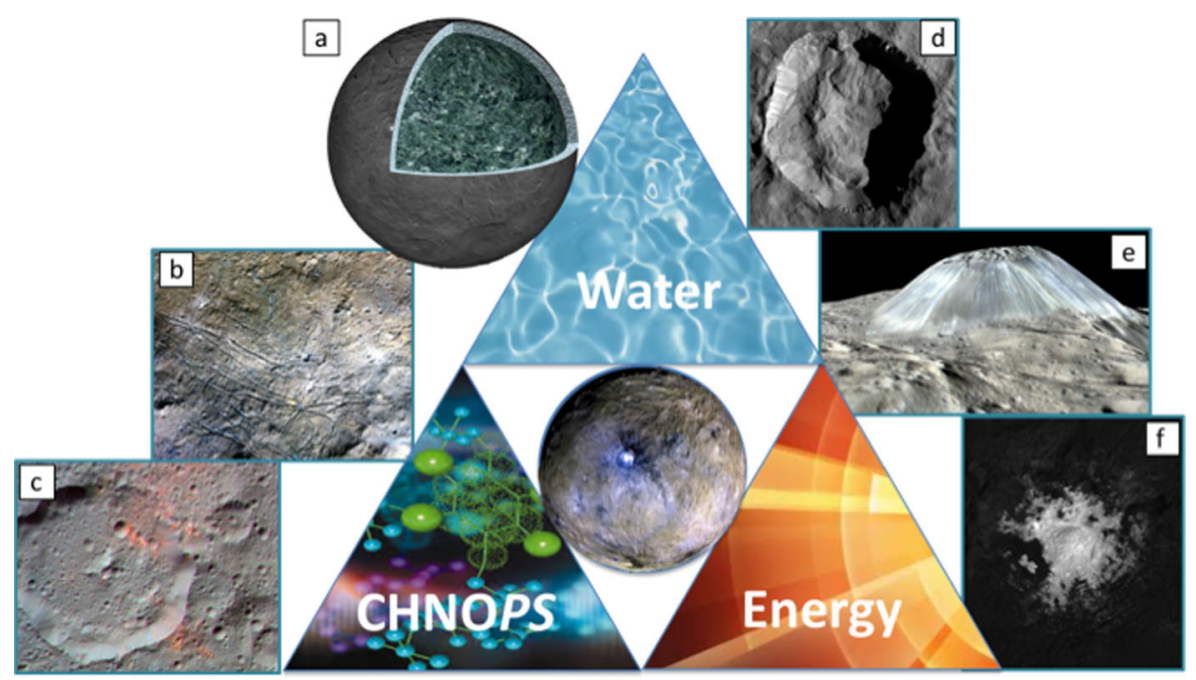

Fig. 6 Summary of Dawn's observations of Ceres addressed in the text. (a) Geophysical data confirmed the abundance of water ice and the need for gas and salt hydrates to explain the observed topography and crustal density. (b) Various types of carbonates and ammonium chloride have been found in many sites across Ceres' surface (e.g., salts exposed on the floor of Dantu crater). (c) Ernuter crater $(\sim 52 \mathrm{~km}$, above) and its area present carbon species in three forms (reduced in CxHy form, oxidized in the form of carbonates and intermediate as graphitic compounds). (d) Ceres shows extensive evidence for water ice in the form of ground ice and exposure via mass wasting and impacts (Left: Juling crater, $\sim 20 \mathrm{~km}$ ). (e) Recent expressions of volcanism point to the combined role of radiogenic heating and low-eutectic brines in preserving melt and driving activity (Left: Ahuna Mons, $\sim 4.5 \mathrm{~km}$ tall). (f) Impacts could create local chemical energy gradients in transient melt reservoirs throughout Ceres' history (Left: Cerealia Facula, $\sim 14 \mathrm{~km}$ diameter). (Figure reprinted from [16]; credit for individual images: NASA/JPL/Caltech/IAPS/MPS/DLR/INAF/ASI)

- Geologically active with minerals present only on Earth and Enceladus

- Closest and most accessible dwarf planet with volcanism and hydrothermal activity

- Ammonia-bearing hydrated minerals, water ice, carbonates, salts, and organic matter make a complex chemical environment that could favour prebiotic chemistry

\subsection{The need for Ceres samples}

Vesta has now joined Mars and the Moon as the best understood extraterrestrial bodies, due at least in part to the fact that we have samples of all of them to study in the laboratory. Ceres can join that group with a future sample return.

Some exploration and scientific arguments for a Ceres sample return are:

- Although carbonaceous chondrites provide the best analog for Ceres [69], we have no meteorites from Ceres. The value of having samples for proper calibration of flight instruments and rigorous interpretation of remote sensing data is illustrated by studies of Vesta, Mars, and the Moon [51]. 
- Global spectral mapping of Ceres by Dawn's VIR demonstrates that its surface is covered almost everywhere by the same assemblage (ammoniated clay, serpentine, carbonate, and a darkening agent), but in slightly differing proportions [3]. Thus, a representative regolith sample can be collected from nearly any location on the surface.

- The ice table begins below several meters depth near the equator, and approaches the surface at higher latitudes [81]. Sampling the regolith in the equatorial region would thus not require cryogenic collection and return.

- We have never before sampled an ocean world, and Ceres could be the most reachable target. There is geophysical evidence for Ceres being an ocean world [16], and our understanding of its alteration is based on the detection of a few minerals. Other phases in minor proportions in a returned sample could constrain the conditions of alteration. It is also likely that Ceres' regolith contains some amorphous phases that could not be characterized by remote sensing.

- There is presently some controversy about the extent to which Ceres' surface has been contaminated by exogenic chondrite impactors. These could be recognized and quantified by petrologic examination of a returned sample.

- Ceres' chronology is based on crater size distribution analysis, which is dependent on the reference impactor flux [66], leaving the absolute ages uncertain. Radiometric dating of a sample from a mapped geologic unit [121] could help anchor Ceres' chronology and the impactor flux in the asteroid belt.

- At several locations on Ceres, notably Ahuna Mons and Occator Crater, brines have recently erupted and deposited salts (sodium-carbonate, ammonium chloride, plus more phases that have not been identified [12, 24, 113, 114, 123]. Small quantities of these phases, which provide important constraints on the nature of subsurface fluids, may be present in regolith samples close to such outcrops.

- Organic matter discovered at one location on Ceres [25] suggests that it should be widely distributed in lesser amounts. The presence of carbon all across Ceres' surface with abundances greater than in CI chondrites suggests a widespread water alteration process associated with organic chemistry [67, 82]. Understanding the organic component on Ceres has important implications for prebiotic chemistry and astrobiology.

- We do not know whether Ceres formed near its present location in the asteroid belt or in the giant planet region and was later scattered into the main belt by giant planet migration and resonances. Measurement of isotopes of $\mathrm{H}, \mathrm{C}$, and $\mathrm{N}$ will place constraints on the origin of water and organics. Isotopes of oxygen, chromium, titanium, etc. [104] can place Ceres into its proper formation setting, as it has for other bodies for which we have samples.

- Comets from the outer Solar System have long been suspected as the source of Earth's water [78]. However, while a recent deuterium-to-hydrogen $(\mathrm{D} / \mathrm{H})$ ratio measurement for a comet is compatible with terrestrial ocean water [42], most comet D/H measurements are not [2]. Meanwhile, dynamical studies [76] indicate that large quantities of Earth's water could have been supplied by objects from the region of the Solar System coinciding with the present-day outer asteroid belt. If Ceres can be determined to have formed in situ, a $\mathrm{D} / \mathrm{H}$ ratio (in addition to other isotopic ratios) measured for Ceres would help to assess the 
plausibility of the main asteroid belt as a source of the terrestrial water that is so critical to the rise of life on Earth.

\section{Mission scenarios}

\subsection{Overview}

If we follow the long-term trend in the development of scientific missions to the Moon and Mars, respectively, and that of asteroidal exploration, we would probably reach the conclusion that they might likely converge on a large-scale international space program of Ceres. NASA's Dawn mission has yielded many exciting results, but the scientific observations were limited to just three remote-sensing instruments, namely the framing camera, the visible and near-infrared mapping spectrometer, the gamma-ray spectrometer/neutron detector, as well as gravity science. A more comprehensive payload on future orbiter(s) is needed for a full characterization and understanding of the surface and atmospheric/exospheric environment of Ceres. In addition, just like in the case of lunar or Mars exploration, different types of platforms such as lander and rovers might be required to address astrobiology objectives. A sample return mission might be regarded as the final step. Furthermore, the waterand organic-rich environment of Ceres makes it an attractive candidate location for future research stations in support of deep space exploration.

As implied by "Dawn", the name of the first mission to Ceres, and the name of the present mission proposal "GAUSS", the in-depth exploration of Ceres should be viewed in terms of detailed investigations of the genesis and evolution of the asteroid belt and the Solar System. The contribution of future in-situ and sample return exploration of Ceres in the context of studying the Solar System ocean worlds and their habitability is illustrated in Fig. 7. It can therefore be envisaged that besides orbiter observations, a set of lander(s) and rover(s) would be needed. It is also possible that this program can be composed of a series of missions over several decades to address different specific scientific questions.

Even though our main focus is to initiate the planning of an ESA-L-class-level sample return mission, it could be streamlined to a Rosetta/Philae lander style mission for cost reasons and expediency. The cost envelope might hence be fitted within the budget of an M-class project depending on the mission component to be chosen. Similar consideration would probably be pursued by CNSA also if it agrees to support a joint assessment study. It is important to emphasize that a sample return mission might still be possible with the participation of additional national agencies, besides CNSA and ESA as postulated here. Possible mission scenarios are summarized in Table 1.

In the scenario of a cryogenic sample return mission, GAUSS will first perform a high-resolution global remote sensing investigation, characterizing the geophysical and geochemical properties of Ceres. Candidate sampling sites will then be identified, and observation campaigns will be run for an in-depth assessment of the candidate sites. Once the sampling site is selected, a lander will be deployed on the surface to collect samples and return them to Earth in cryogenic conditions that 


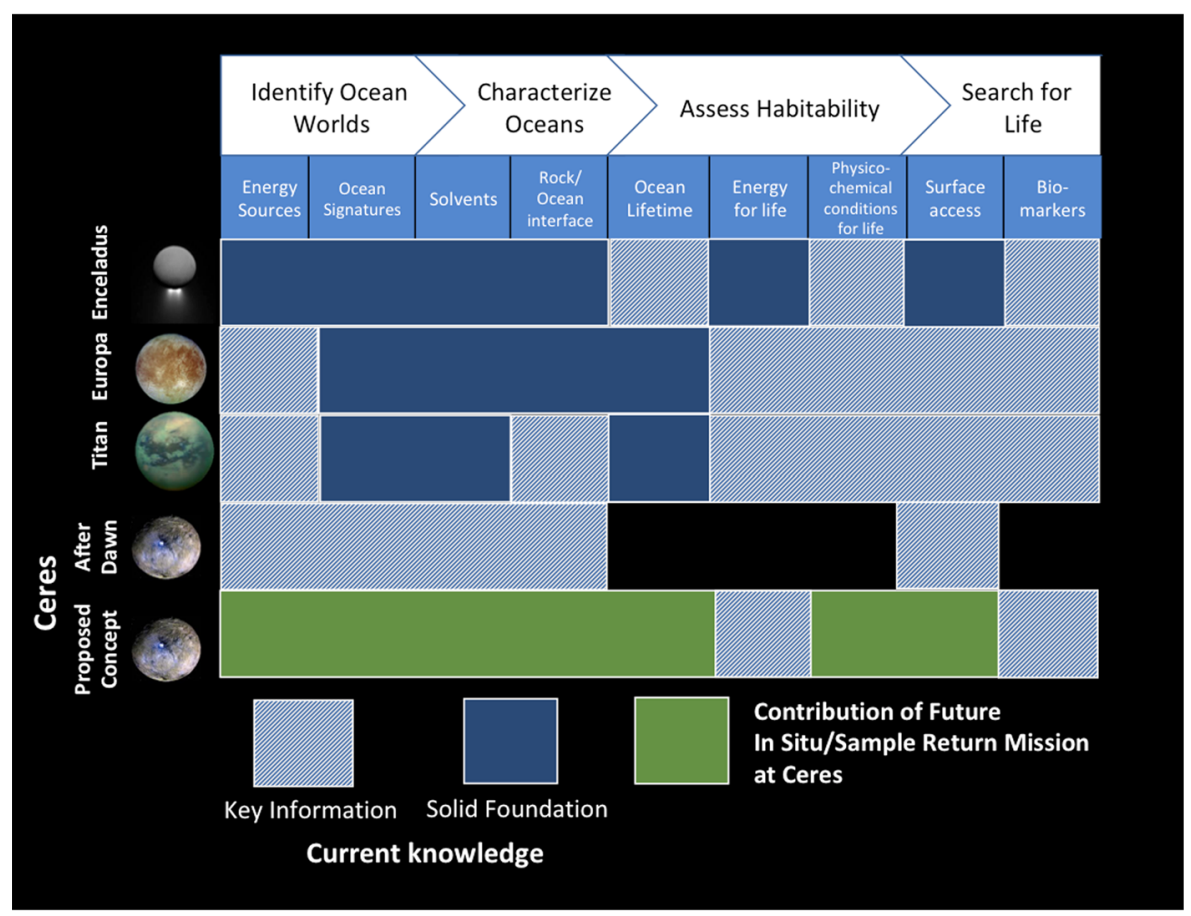

Fig. 7 The contribution of future Ceres in-situ and sample return mission to the exploration of the Solar System ocean worlds and their habitability (Based after [44])

preserve the volatile and organic composition as well as the original physical state enabled by state of the art technologies.

The scientific goals and related measurement objectives of Project GAUSS are summarized in the traceability matrix (Table 2). The four scientific goals focus on understanding: the origin of Ceres, its evolution and current state, its habitability, and its connection to the carbonaceous meteorite collections. The results from these investigations will have direct implications on our understanding of the evolution of the Solar System and in particular of the icy satellites.

Table 1 Summary of mission scenarios

\begin{tabular}{lll}
\hline Mission type & Mission component & Mission class \\
\hline Sample return & $\begin{array}{l}\text { Orbiter+lander+return L-class, or M-class with } \\
\text { capsule }\end{array}$ & $\begin{array}{l}\text { significant contribution } \\
\text { from CNSA }\end{array}$ \\
Landing/Roving & $\begin{array}{l}\text { Orbiter+lander } \\
\text { hopper/ rover }\end{array}$ & $\begin{array}{l}\text { M-class with possible lan- } \\
\text { der/rover contribution by } \\
\text { CNSA }\end{array}$ \\
\hline
\end{tabular}


Table 2 Traceability matrix for Project GAUSS

\begin{tabular}{|c|c|c|}
\hline Scientific goal & Measurement objective & Instrument \\
\hline \multicolumn{3}{|c|}{$\begin{array}{l}\text { The origin and transportation of water and other volatiles in the inner Solar } \\
\text { System: Where does Ceres come from? }\end{array}$} \\
\hline Ceres chronology & $\begin{array}{l}\text { Crater counting, radiomet- } \\
\text { ric dating of samples }\end{array}$ & $\begin{array}{l}\text { Sample analysis, camera, topo- } \\
\text { graphic camera }\end{array}$ \\
\hline $\begin{array}{l}\text { Connection between Ceres } \\
\text { composition and pre-solar } \\
\text { materials }\end{array}$ & $\begin{array}{l}\text { Stable isotopes of oxygen, } \\
\text { chromium, titanium }\end{array}$ & $\begin{array}{l}\text { Sampling mechanism, microscopic } \\
\text { camera }\end{array}$ \\
\hline Volatile inventory on Ceres & $\begin{array}{l}\text { Surface and subsurface } \\
\text { water, other volatile } \\
\text { species including } \mathrm{Na}, \mathrm{K} \text {, } \\
\mathrm{S}, \mathrm{Cl} \text {, etc. }\end{array}$ & $\begin{array}{l}\text { Sampling mechanism, Near- } \\
\text { Infrared spectrometer (NIR), } \\
\text { Thermal Infrared spectrometer } \\
\text { (TIR), Active Particle-induced X- } \\
\text { ray Spectrometer (APXS), Gamma } \\
\text { Ray Spectrometer (GRS) }\end{array}$ \\
\hline $\begin{array}{l}\text { Contamination from } \\
\text { exogenous materials on }\end{array}$ & $\begin{array}{l}\text { Surface mineralogy, petro- } \\
\text { logical units in samples }\end{array}$ & $\begin{array}{l}\text { Sampling mechanism, camera, } \\
\text { NIR, TIR, microscopic camera }\end{array}$ \\
\hline
\end{tabular}

Ceres and their roles in the evolution of Ceres

Physical properties and internal structure of Ceres: What do ice dwarf planets look like?

Structure of the nearsubsurface and deep interior, and the implications to the differentiation and aqueous alteration processes of Ceres

Geological processes, in particular current and past cryovolcanism

The existence and characteristics of Ceres' exosphere
Surface mineralogy, elemental abundance, gravity
Imaging, NIR, TIR, seismology, radar, subsurface science package
Topography and morphology, gravity

Gas species around Ceres
Imaging, lidar, radar, radio science, seismometer

Gas chromatography-mass spectrometer (GC-MS), Ion and mass spectrometer, UV spectrometer, dust detector

The astrobiological implications of Ceres: Was it habitable in the past and is it still today?

Existence of liquid water inside Ceres, its extent, distribution, and depth

Redox condition on Ceres, the existence and forms of oxidants

Abundance, sources and sinks, and chemical forms of life-forming elements; source of terrestrial water
Topography, morphology, gravity

Elemental abundance, forms, and isotopic ratios of $\mathrm{O}, \mathrm{S}, \mathrm{Cl}, \mathrm{N}$

Elemental abundance, forms, and isotopic ratios of C, H, N, O, S
Camera, radar, lidar, topographic camera, radio science, seismometer, subsurface science package

Sampling mechanism, NIR, TIR, APXS, GRS

Sampling mechanism, NIR, TIR, APXS, GRS 
Table 2 (continued)

\begin{tabular}{|c|c|c|}
\hline Scientific goal & Measurement objective & Instrument \\
\hline $\begin{array}{l}\text { Inventory and com- } \\
\text { position of organic } \\
\text { compounds, their origins } \\
\text { and evolutions }\end{array}$ & $\begin{array}{l}\text { Existence, abundance, and } \\
\text { types of organic materials }\end{array}$ & $\begin{array}{l}\text { Sample mechanism, NIR, } \\
\text { TIR }\end{array}$ \\
\hline \multicolumn{3}{|c|}{$\begin{array}{l}\text { Mineralogical connection between Ceres and the collections of primitive mete- } \\
\text { orites: Where are Ceres meteorites? }\end{array}$} \\
\hline $\begin{array}{l}\text { Thermal metamorphism } \\
\text { and aqueous alteration } \\
\text { history of Ceres }\end{array}$ & $\begin{array}{l}\text { Mineralogy, petrological } \\
\text { characterization, isotope } \\
\text { ratios }\end{array}$ & Sampling mechanism, NIR, TIR \\
\hline $\begin{array}{l}\text { Fractionation of elements } \\
\text { and the geochemical pro- } \\
\text { cesses }\end{array}$ & $\begin{array}{l}\text { Mineralogy, elemental } \\
\text { abundance, isotope ratios }\end{array}$ & Sampling mechanism, NIR, TIR \\
\hline
\end{tabular}

\subsection{Candidate sites for in-situ investigation and sample return}

The geomorphological features on the surface of Ceres are connected to its interior and formed through recent geological processes (Fig. 8). The mantle of Ceres is composed of hydrated minerals, and may be enriched in organics and high-density, low melting point organic matter in localized areas. The $\sim 40 \mathrm{~km}$ thick crust above the mantle is composed of the original ocean materials, salts, carbonates, and brine. Brine reservoirs existed in the recent past and drove volcanic activity. Ahuna Mons might be an extrusion feature of briny mud and organics from the upper mantle [95]. The surface layer of crust is covered by a mixture of infalls, salts, and organics. Cerealia Facula is covered by salts that are left behind after subsurface brine was accessed by impact-produced fractures and reached the surface through the cracks and eventually evaporated [87]. The Haulani crater is one of the youngest impact craters, associated with bright blue rays of ejecta that are freshly exposed crustal materials and could represent the original ocean materials [113].Therefore, the Dawn observations of Ceres indicate these sites carry profound scientific implications serving the scientific goals identified for Project GAUSS for in-situ investigations and sample returns (Fig. 9).

\subsection{Proposed payload}

Only three instruments and gravity science were carried by Dawn. A more comprehensive payload would be needed to characterize Ceres itself and its atmospheric and space environment. This is especially true with the detection of water plume activity by Herschel [59]. Similar to the Cassini measurements at Enceladus, repeated flythrough of the gas plume would allow the identification of the chemical composition and isotopic ratios of the gas molecules, thus providing a probe to the nature of the subsurface lake/ocean. The proposed payload and their heritage are summarized in Table 3. It is assumed that some of the scientific instruments will have been successfully developed for CNSA's Tianwen-1 mission to Mars and the China Asteroid 


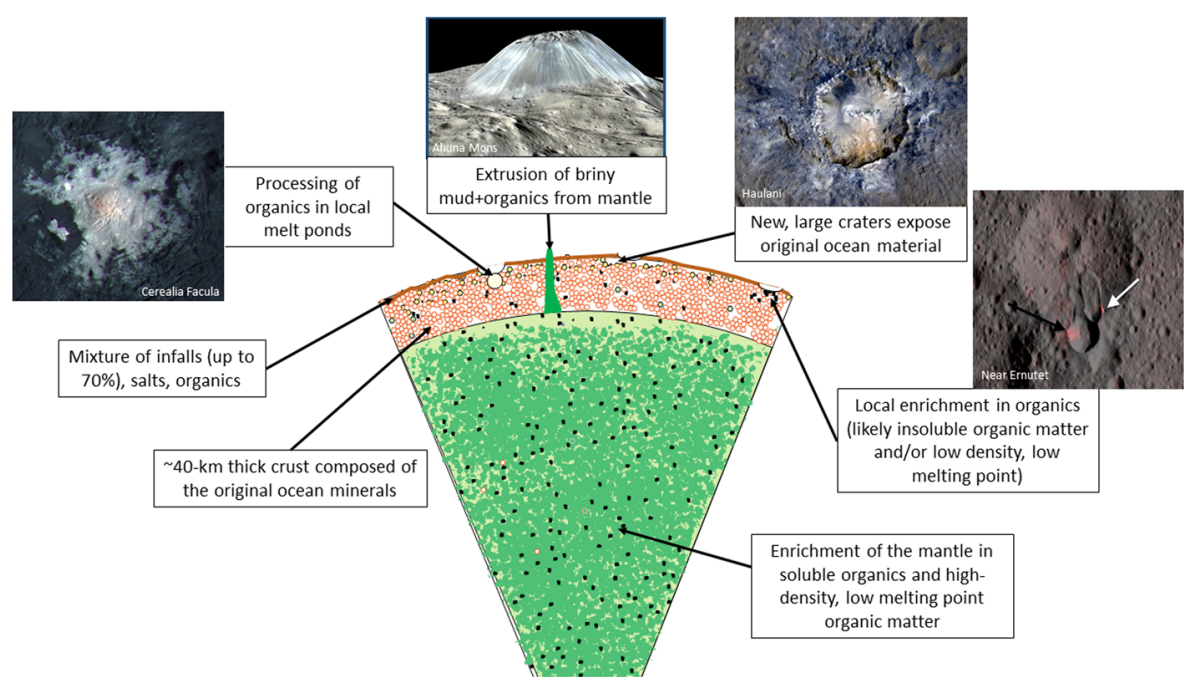

Fig. 8 Surface geomorphological features on Ceres and their connections to the interior structure (image credit: NASA/JPL/Caltech/IAPS/MPS/DLR/INAF/ASI)

Explorer (CAEX) to small bodies. By the same token, the sampling and reentry technology are also assumed to be derived from China's sampling missions to the Moon, Mars, and asteroids. The instrumentation on the Philae lander on ESA's Rosetta, the Rosalind Franklin Rover (RFR) of ExoMars, the Yutu rovers of Chang'e missions, and the Zhurong rover of the Tianwen-1 mission, will provide strong preparation and heritage for the Ceres rover.

It is important to emphasize the astrobiological significance of the in-situ exploration of geological landmarks of special interest. These include the Ernutet crater and the Occator crater. To achieve high confidence in detecting organic materials on the surface of Ceres and assess their nature, the mid-IR range $2-12 \mu \mathrm{m}$ turns out to be crucial at the spatial resolution that could be achieved by a spacecraft in orbit around Ceres, not necessarily with imaging capabilities. Covering this sensitivity range would allow:

- Complementing surface mineralogy as derived by VIR in the overall $0.4-5.1 \mu \mathrm{m}$ spectral range by using thermal emission spectroscopy from $2-12 \mu \mathrm{m}$, determining the specific class of compounds responsible for the organics-rich area observed close to crater Ernutet, and shedding light on its origin (endogenous vs. exogenic). This spectral range would ultimately provide the capability to greatly expand our inventory of astrobiologically important compounds, and to remotely sense complex organics.

- Assess any potential ongoing activity and occurrence of organics, particularly on top of Cerealia Facula in crater Occator.

- Perform an in-depth characterization of the 34-km crater Haulani, one of the youngest geologic features on Ceres and home to the most prominent thermal signature observed on the entire surface of the dwarf planet [113, 114]. 


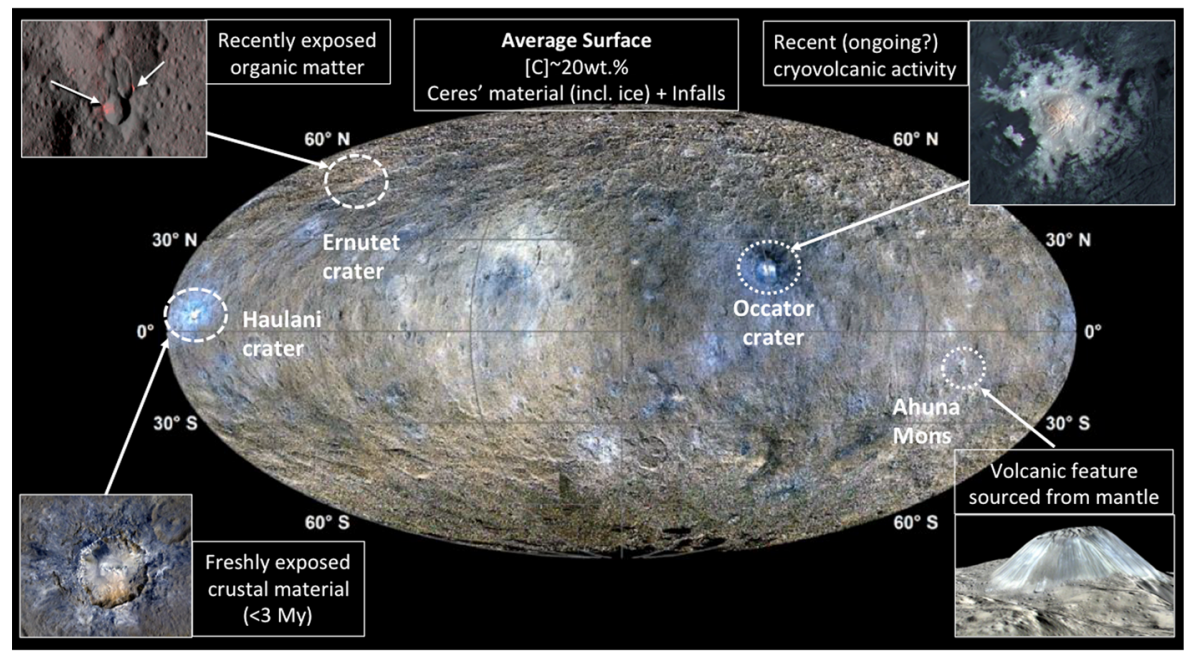

Fig. 9 Candidate sites on Ceres for in-situ and/or sample return. The Ceres basemap is a composite color map of Ceres generated by Dawn Framing Camera images with R, G, B $=960,550,440 \mathrm{~nm}$, respectively (from Castillo-Rogez et al, Pre-decadal survey, image credit: NASA/JPL/Caltech/IAPS/MPS/DLR/INAF/ASI)

- Retrieve surface temperatures, grain size, porosity and surface roughness using thermal emission from $2-12 \mu \mathrm{m}$, thus accessing temperature values below $180 \mathrm{~K}$ with high accuracy, which were precluded to Dawn/VIR.

- Ultimately characterize the best landing site for a surface element in terms of composition and roughness (regolith depth).

The Fourier Transform Spectrometer (FTS) working principle is based on the Michelson interferometer, which is an infrared spectrometer suitable for covering a broad spectral range from the near infrared to the mid infrared with constant, high spectral resolution (typically up to $1-2 \mathrm{~cm}^{-1}$ ). FTSs simultaneously detect light varying the optical path difference (OPD) and encoding the signal at each wavelength with a cosine modulation at a frequency proportional to the wavenumber $\nu=1 / \lambda$. This interferogram is then transformed into a spectrum, i.e., from the optical path domain to the optical frequency domain. The OPD could be produced by rotating a double pendulum system around its axis, rather than translating the moving mirror along a linear direction like in the classical Michelson interferometer. Compared to the first generation of FTS (e.g. PFS on board the ESA Mars Express spacecraft), in recently upgraded versions have been proposed that offer a significant reduction in mass and size, adopting innovative technical solutions.

\subsection{Trajectory design}

Considering the fact that the target asteroid is located between the orbits of Mars and Jupiter (the semi-major axis of Ceres is about $2.768 \mathrm{au}$ ), in the transfer design we take advantage of a gravity assist of Mars in order to reduce the required fuel 
Table 3 Strawman payload on the Orbiter and the Lander

\begin{tabular}{|c|c|c|}
\hline & Payload name & Heritage \\
\hline \multirow[t]{12}{*}{ Orbiter } & Wide-angle and narrow-angle camera & Chang'e 1-3, Dawn, Rosetta, BepiColombo \\
\hline & Infrared imaging spectrometer & Dawn, Rosetta, Chang'e-4, CAEX \\
\hline & Fourier Transform Spectrometer & Mars Express, ExoMars \\
\hline & Thermal mapper & CAEX \\
\hline & Ultraviolet imaging spectrometer & Chang'e-3, MMX \\
\hline & GRS & Change'e $1-2$ \\
\hline & Long-wavelength radar & CAEX \\
\hline & Lidar & BepiColombo \\
\hline & Dust detector & Rosetta, CAEX \\
\hline & Ion and mass spectrometer & Rosetta, CAEX \\
\hline & Particles and Fields package & Rosetta, CAEX \\
\hline & Radio Science & Rosetta \\
\hline \multirow[t]{8}{*}{ Lander } & Topographic camera system & Chang'e 3-4; Mars rovers; Philae \\
\hline & Microscopic camera & Philae, CAEX, CLUPI (ExoMars) \\
\hline & APXS & Chang'e 1-3 \\
\hline & GRS & Chang'e 1-2 \\
\hline & Laser-induced breakdown spectroscopy & Tianwen-1, Curiosity \\
\hline & GC-MS & Philae, CAEX \\
\hline & Subsurface science package & Philae, CAEX \\
\hline & Active seismometer & Insight, Chang'e 7 \\
\hline
\end{tabular}

consumption. In the preliminary transfer scenario, the sequence is given as follows: (a) launching from the Earth; (b) Mars' gravity assist; (c) rendezvous with Ceres; (d) returning to the Earth. The launch window is assumed to be after January 1st, 2035. The planar transfer trajectory is reported in Fig. 10 and the associated parameters are provided in Table 4.

\subsection{Technological developments required}

Here we discuss the key technological requirements and main challenges for a Ceres sample return mission.

Flight dynamics It is assumed, that by the time of the Voyage 2050 programme, the capacity of launch vehicles poses no challenge for sending a spacecraft with a lander and re-launch system to Ceres. Ceres does not have an atmosphere that exhibited any detectable dynamical effects on the Dawn spacecraft at altitudes down to $35 \mathrm{~km}$. Therefore, an atmospheric entry system is not needed to land on Ceres. The descent and landing system for Ceres can be derived from the already mature Chinese Chang'e 3 and 4 descent and landing system. The technology for a re-launch system already exists since the Apollo era, and a similar system is already in development 


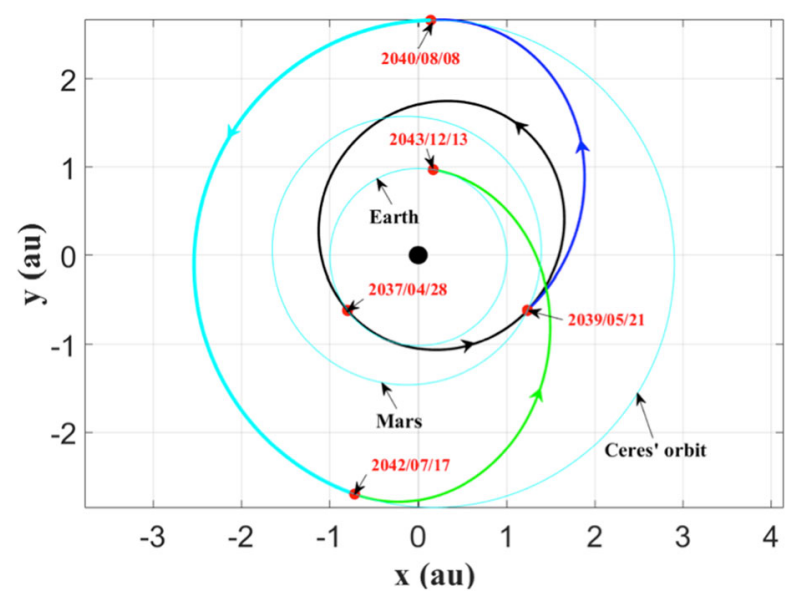

Fig. 10 The transfer trajectory for Ceres' sample return mission. In the transfer scenario, a Mars' gravity assist is taken into consideration. The probe launches from the Earth on April 28th, 2037. The arc from the Earth to Mars is shown in black line, the arc from Mars to the target asteroid is given in blue line, the rendezvous segment is presented in cyan line, and the return trajectory from the asteroid to the Earth is marked in green line. The critical points of time are represented by red dots

for the various Mars sample return missions under study (see Visions and Voyages, the Planetary Science Decadal Survey 2013-2022 report, National Research Council of the National Academies). The escape velocity from Ceres' surface of about 0.5 $\mathrm{km} / \mathrm{s}$ is much lower than that of the Moon $(2.4 \mathrm{~km} / \mathrm{s})$ and Mars $(5 \mathrm{~km} / \mathrm{s})$. Therefore, the re-launch system from Ceres' surface for sample return can be much smaller and light-weight than those for the Apollo system (which also had a complicated lifesupport system) or any Mars sample return system in development. It can be expected that the needed technology will have been developed and matured by the 2030-2040 timeframe, and should be much more efficient (small and light-weight) than the currently available system and hence will require a less powerful launch vehicle than required by present technology. Earth re-entry is already mature and is not expected to pose any challenge. In addition, an ion propulsion system, like the one used by the Dawn spacecraft should be much more efficient.

Sampling on Ceres One or more approaches need to be developed for sampling the surface layer of Ceres. Sampling on Ceres is different from that on the Moon or Mars, which have relatively strong surface gravity and don't require an anchoring mechanism. Ceres sampling would also be different from that on small asteroids such as the targets of JAXA's Hayabusa and Hayabusa 2, and NASA's OSIRIS-REx, which have loose regolith and micro-gravity allowing for a touch-and-go approach. With its surface gravitational acceleration of $\sim 0.27 \mathrm{~m} / \mathrm{s}^{2}$, a robust anchoring mechanism may be required for sampling on Ceres, depending on the sampling system. A drilling system may also be needed to drill into the relatively strong rock-ice-salt mixture with a density of $\sim 1.3 \mathrm{~g} / \mathrm{cm}^{3}$ [29]. A drilling depth of decimetres, but less than one metre is likely needed in order to reach the water-ice rich layers or subsurface ice 
Table 4 Parameters of the interplanetary trajectory for the Ceres sample return mission

\begin{tabular}{lllr}
\hline Time points & Velocity impulse & \\
\hline Launch & April 28, 2037 & Hyperbolic excess velocity $\left(V_{\infty}\right)$ & $4.961 \mathrm{~km} / \mathrm{s}$ \\
Mars' gravity assist & May 21, 2039 & Deep space maneuver at perimartian $\left(\Delta V_{M}\right)$ & $1.981 \mathrm{~km} / \mathrm{s}$ \\
Rendezvous with Ceres August 8, 2040 & Braking velocity for rendezvous with Ceres $\left(\Delta V_{f}\right) 5.400 \mathrm{~km} / \mathrm{s}$ \\
$\begin{array}{l}\text { Departure from Ceres } \\
\text { Earth re-entry }\end{array}$ & July 17, 2042 & Accelerating velocity for departing from Ceres & $4.813 \mathrm{~km} / \mathrm{s}$ \\
& & &
\end{tabular}

table at high latitudes [81]. Technologies currently being developed for landing on and sampling asteroids might provide the necessary heritage for project GAUSS, e.g., technologies being developed for the Chinese small body mission [125]. The various sampling mechanisms and collection system for Mars sample return currently in use $[65,122]$ are good references for the future development of a Ceres sample return system. Note that ESA and NASA signed a statement of intent on Mars sample return.

Cryogenic sample collection, containment, and curation The idea of cryogenic sample return from icy Solar System bodies has been around for a long time. The Rosetta mission was originally designed to return samples from a comet at cryogenic temperature [49]. This is probably the most challenging part of the entire project. To preserve the volatile and organic compounds in their original status, samples should not be thermally or aqueously altered during the collection, storage and transport, and they should be shielded from contamination by terrestrial volatiles upon return. Therefore, the samples need to be collected and sealed in the containers under cryogenic temperature and overpressure. A temperature of $<\sim 170 \mathrm{~K}$ is required, and the original temperature of subsurface samples (probably $\sim 140 \mathrm{~K}$; $[43,99]$ ) is desired, throughout the entire collection, return, and curation process for preserving water ice. The sample return capsule should have a number of separated and individually sealed containers that can be overpressured to prevent the loss of volatiles such as $\mathrm{H}_{2} \mathrm{O}, \mathrm{HCN}$, S-bearing species, and cyanides [17, 18].

\subsection{Planetary protection}

As revealed by the Dawn observations, the surface of Ceres is organic rich, with certain areas exhibiting higher concentration (e.g. the Ernutet crater; [25]). Furthermore, multiple regions on Ceres show signs of recent or even ongoing brine-driven activity that could expose fresh materials from Ceres' interior. Although it is unlikely that the organic matter on Ceres is of biological origin, it is required to assess planetary protection strategies for any future lander or sample return mission to Ceres. Based on the current guidelines for planetary protection [39] and synthesized analyses of the Dawn results, we summarize in Table 5 preliminary considerations on planetary protection for different types of mission to Ceres. 
Table 5 Planetary protection categories for different types of missions to Ceres

\begin{tabular}{llll}
\hline Mission type & Category & Note \\
\hline Lander/Rover & Most part of the surface & $\mathrm{II}^{*}$ & $\begin{array}{l}\text { Like Dawn, the mission } \\
\text { is at least Category III if } \\
\text { it includes a Mars gravity } \\
\text { assist. }\end{array}$ \\
Sample return & $\begin{array}{l}\text { Occator faculae } \\
\text { Different possible sites, } \\
\text { including } \\
\text { areas }\end{array}$ & V IV & $\begin{array}{l}\text { Determination on the } \\
\text { sub-category of being } \\
\text { "restricted" or } \\
\text { stricted" depends } \\
\text { location. }\end{array}$ \\
\end{tabular}

Further information to be found in Castillo-Rogez, J. C. et al. "Planetary Protection Requirements for Future Exploration of Ceres-State of Understanding after the Dawn Mission" (in revisions)

\section{International context}

We note that the ESA roadmap to Ceres needs to be constructed within an international context. NASA has recently selected in situ exploration and sample return concepts at Ceres to be studied in preparation for the 2023-2032 planetary science decadal survey [14]. That study will cover a range of architectures with a focus on long-range mobility, sample acquisition, and return to Earth. CNSA has also expressed interest in a mission to Ceres beyond its exploration of the Moon, Mars and small bodies [127]. It is, therefore, essential for ESA to explore cooperative opportunities through joint assessment studies with international agencies.

This mission concept came about during a round-table discussion at the 4th Lunar and Deep-Space Exploration International Conference between July 22 and 24, 2019, in Zhuhai, China. One important objective of this proposal is therefore to promote scientific cooperation of the Chinese planetary science community with its European counterpart, taking advantage of the momentum of CNSA and the spirit of ESA's "Voyage 2050 Initiative". It might begin with a joint assessment study co-sponsored by both agencies or their representatives. The assessment should identify the scientific objectives, technical requirements, mission architecture, and possible division of responsibilities and authorities.

The extensive orbital measurements of the Dawn mission can be used as a robust basis for planning this large scale mission. Because of the efforts of several agencies including JAXA, NASA, and ESA over the last two decades, the exploration of asteroids has become a major component of deep space missions. On the one hand, sample return missions and the related technologies have been championed by JAXA that have successfully executed the Hayabusa mission to the S-type asteroid Itokawa and the Hayabusa2 mission to the C-type asteroid Ryugu. This line of approach will be extended to a sample return mission (Mars Moon Explorer or MMX) to the Martian moon Phobos and likely a sample return mission to a Jovian Trojan asteroid [77]. Following the Dawn mission, NASA has demonstrated its strong interest in asteroid in-situ exploration by performing the OSIRIS-REx sample-collection project to 
the B-type asteroid Bennu, and developing two more asteroid rendezvous missions, one to the large M-type asteroid 16 Psyche, and the other one (Lucy) to a number of Jovian Trojans (flybys). The Chinese space agency, CNSA, has recently issued an AO calling for instrument proposals and international cooperation for its first asteroid sample return mission to a near-Earth asteroid, followed by a rendezvous mission with a main-belt comet [126]. There is also an ongoing assessment study for a sample return mission to an inner-belt E-type asteroid. In parallel to this heightened level of activities by JAXA, NASA and CNSA, ESA has approved the daring "Comet Interceptor" project on the heel of the Rosetta/Philae mission.

If the above set of planned (or proposed) space projects can be successfully carried out, then the first phase of reconnaissance/sample-return missions to major phenotypes of small asteroids and short-period comets-as a global enterprisewould be completed by 2030-2035. It is also expected that the basic knowledge gained and technologies introduced would be applied to the next phase of asteroidal exploration-beginning around 2030, if not earlier, with the goal to address the need of planetary defense against asteroid impact hazard, and asteroidal mining for commercial reasons.

The lunar samples returned by the Apollo missions half a century ago continue to spur cutting-edge science. We believe that Ceres should be viewed as the next frontier space laboratory to extend our knowledge of the Solar System much further beyond.

The GAUSS Team (continued) John Carter (Institut d'Astrophysique Spatiale), Grégoire Danger (Aix Marseille Univ, CNRS), Julia de Leon (Instituto de Astrofísica de Canarias), Jörn Helbert (DLR Institute of Planetary Research), Xiyun Hou (Nanjing University), Hauke Hussmann (DLR Institute of Planetary Research), Katherine Joy (University of Manchester), Tomas Kohout (University of Helsinki), Alice Lucchetti (INAF-Astronomical Observatory of Padova), David Mimoun (Université de Toulouse), Olga Muñoz (Instituto de Astrofisica de Andalucia), Jose Luis Ortiz (Instituto de Astrofisica de Andalucia), Maurizio Pajola (INAF-Astronomical Observatory of Padova), Antti Penttilä (University of Helsinki), Frank Preusker (DLR Institute of Planetary Research), Ottaviano Ruesch (University of Münster), Pablo Santos-Sanz (Instituto de Astrofisica de Andalucia), Nico Schmedmann (University of Münster), Nicole Schmitz (DLR Institute of Planetary Research), Katrin Stephan (DLR Institute of Planetary Research), Guneshwar Thangjam (NISER), Josep M. Trigo-Rodríguez (Institute of Space Sciences, CSIC-IEEC), Cecilia Tubiana (INAF-IAPS), Vassilissa Vinogradoff (Aix Marseille University, CNRS), Liangliang Yu (Macau University of Science and Technology), Francesca Zambon (INAF-IAPS), Yuhui Zhao (Purple Mountain Observatory, CAS)

Acknowledgements The early concept of the white paper has benefitted from various discussions with Professor Adam Showman, who sadly passed away in March 2020. His contribution and influence are gratefully acknowledged and sorely missed. Part of this work has been carried out at the Jet Propulsion Laboratory, California Institute of Technology, under a contract with the National Aeronautics and Space Administration (80NM0018D0004). J.-Y.L. acknowledges partial support from the Solar System Exploration Research Virtual Institute 2016 (SSERVI16) Cooperative Agreement (grant NNH16ZDA001N), SSERVI-TREX to the Planetary Science Institute. J.A. acknowledges support from the European Research 
Council Starting Grant 757390 (CAstRA). A.J.C. and G.H.J. acknowledge support from the STFC consolidated grant to UCL-MSSL STS0002401. P. Santos-Sanz, and R. Duffard acknowledges financial support by the Spanish grant AYA- RTI2018-098657-J-I00 'LEO-SBNAF' (MCIU/AEI/FEDER, UE). J.L. Ortiz, P. Santos-Sanz, and R. Duffard acknowledge financial support from the State Agency for Research of the Spanish MCIU through the 'Center of Excellence Severo Ochoa' award for the Instituto de Astrofísica de Andalucía (SEV-2017-0709). J.M.T-R. acknowledges support from the Spanish Ministry of Science and Innovation (project PGC2018-097374-B-I00). J.M.T-R.'s research has been funded by the research project (PGC2018-097374-B-I00), funded by FEDER/Ministerio de Ciencia e Innovación - Agencia Estatal de Investigación.

Funding Open Access funding enabled and organized by Projekt DEAL.

Open Access This article is licensed under a Creative Commons Attribution 4.0 International License, which permits use, sharing, adaptation, distribution and reproduction in any medium or format, as long as you give appropriate credit to the original author(s) and the source, provide a link to the Creative Commons licence, and indicate if changes were made. The images or other third party material in this article are included in the article's Creative Commons licence, unless indicated otherwise in a credit line to the material. If material is not included in the article's Creative Commons licence and your intended use is not permitted by statutory regulation or exceeds the permitted use, you will need to obtain permission directly from the copyright holder. To view a copy of this licence, visit http://creativecommons.org/licenses/by/4.0/.

\section{References}

1. A'Hearn, M.F., Feldman, P.D.: Water vaporization on Ceres. Icarus 98(1), 54-60 (1992). https://doi.org/10.1016/0019-1035(92)90206-M

2. Altwegg, K., Balsiger, H., Bar-Nun, A., Berthelier, J.J., Bieler, A., Bochsler, P., Briois, C., Calmonte, U., Combi, M., De, K.eyser.J., Eberhardt, P., Fiethe, B., Fuselier, S., Gasc, S., Gombosi, T.I., Hansen, K.C., Hässig, M., Jäckel, A., Kopp, E., Korth, A., LeRoy, L., Mall, U., Marty, B., Mousis, O., Neefs, E., Owen, T., Rème, H., Rubin, M., Sémon, T., Tzou, C.Y., Waite, H., Wurz, P.: 67P/ChuryumovGerasimenko, a Jupiter family comet with a high D/H ratio. Science 347(6220), 1261952 (2015). https://doi.org/10.1126/science.1261952

3. Ammannito, E., DeSanctis, M.C., Ciarniello, M., Frigeri, A., Carrozzo, F.G., Combe, J.P., Ehlmann, B.L., Marchi, S., McSween, H.Y., Raponi, A., Toplis, M.J., Tosi, F., Castillo-Rogez, J.C., Capaccioni, F., Capria, MT., Fonte, S., Giardino, M., Jaumann, R., Longobardo, A., Joy, S.P., Magni, G., McCord, T.B., McFadden, L.A., Palomba, E., Pieters, C.M., Polanskey, C.A., Rayman, MD., Raymond, CA., Schenk, PM., Zambon, F., Russell, C.T.: Distribution of phyllosilicates on the surface of Ceres. Science 353(6303), aaf4279 (2016). https://doi.org/10.1126/science.aaf4279

4. Bell, C.P.M., Naylor, T., Mayne, N.J., Jeffries, R.D., Littlefair, S.P.: Pre-main-sequence isochrones - II. Revising star and planet formation time-scales. MNRAS 434(1), 806-831 (2013). https://doi.org/10.1093/mnras/stt1075

5. Bland, M.T., Raymond, C.A., Schenk, P.M., Fu, R.R., Kneissl, T., Pasckert, J.H., Hiesinger, H., Preusker, F., Park, R.S., Marchi, S., King, S.D., Castillo-Rogez, J.C., Russell, C.T.: Composition and structure of the shallow subsurface of Ceres revealed by crater morphology. Nat. Geosci. 9(7), 538-542 (2016). https://doi.org/10.1038/ngeo2743

6. Bottke, W.F., Vokrouhlický, D., Rubincam, D.P., Broz, M.: The Effect of Yarkovsky Thermal Forces on the Dynamical Evolution of Asteroids and Meteoroids, pp 395-408 (2002)

7. Bottke, W.F., Durda, D.D., Nesvorný, D., Jedicke, R., Morbidelli, A., Vokrouhlický, D., Levison, H.F.: Linking the collisional history of the main asteroid belt to its dynamical excitation and depletion. Icarus 179(1), 63-94 (2005). https://doi.org/10.1016/j.icarus.2005.05.017

8. Bottke, W.F., Vokrouhlický, D., Rubincam, D.P., Nesvorný, D.: The yarkovsky and yorp effects: Implications for asteroid dynamics. Annu. Rev. Earth Planet. Sci. 34(1), 157-191 (2006). https://doi.org/10.1146/annurev.earth.34.031405.125154

9. Buczkowski, D.L., Schmidt, B.E., Williams, D.A., Mest, S.C., Scully, J.E.C., Ermakov, A.I., Preusker, F., Schenk, P., Otto, KA., Hiesinger, H., O’Brien, D., Marchi, S., Sizemore, H., Hughson, 
K., Chilton, H., Bland, M., Byrne, S., Schorghofer, N., Platz, T., Jaumann, R., Roatsch, T., Sykes, M.V., Nathues, A., De Sanctis. M.C., Raymond, C.A., Russell, C.T.: The geomorphology of Ceres. Science 353(6303), aaf4332 (2016). https://doi.org/10.1126/science.aaf4332

10. Buczkowski, D.L., Scully, J.E.C., Quick, L., Castillo-Rogez, J., Schenk, P.M., Park, R.S., Preusker, F., Jaumann, R., Raymond, C.A., Russell, C.T.: Tectonic analysis of fracturing associated with occator crater. Icarus 320, 49-59 (2019). https://doi.org/10.1016/j.icarus.2018.05.012

11. Budde, G., Burkhardt, C., Brennecka, G.A., Fischer-Gödde, M., Kruijer, T.S., Kleine, T.: Molybdenum isotopic evidence for the origin of chondrules and a distinct genetic heritage of carbonaceous and non-carbonaceous meteorites. Earth Planet. Sci. Lett. 454, 293-303 (2016). https://doi.org/10.1016/j.epsl.2016.09.020

12. Carrozzo, F.G., De Sanctis, M.C., Raponi, A., Ammannito, E., Castillo-Rogez, J., Ehlmann, B.L., Marchi, S., Stein, N., Ciarniello, M., Tosi, F., Capaccioni, F., Capria, M.T., Fonte, S., Formisano, M., Frigeri, A., Giardino, M., Longobardo, A., Magni, G., Palomba, E., Zambon, F., Raymond, C.A., Russell, C.T.: Nature, formation, and distribution of carbonates on Ceres. Sci. Adv. 4(3), e1701645 (2018). https://doi.org/10.1126/sciadv.1701645

13. Castillo-Rogez, J.: Future exploration of Ceres as an ocean world. Nature Astronomy 4, 732-734 (2020). https://doi.org/10.1038/s41550-020-1181-5

14. Castillo-Rogez, J., et al.: Concepts for the Future Exploration of Dwarf Planet Ceres' Habitability. Planetary Science Journal, in revisions (2021)

15. Castillo-Rogez, J., Neveu, M., McSween, H.Y., Fu, R.R., Toplis, M.J., Prettyman, T.: Insights into Ceres's evolution from surface composition. Meteorit. Planet. Sci. 53(9), 1820-1843 (2018). https://doi.org/10.1111/maps.13181

16. Castillo-Rogez, J.C., Neveu, M., Scully, J.E.C., House, C.H., Quick, L.C., Bouquet, A., Miller, K., Bland, M., De Sanctis, M.C., Ermakov, A., Hendrix, A.R., Prettyman, T.H., Raymond, C.A., Russell, C.T., Sherwood, B.E., Young, E.: Ceres: Astrobiological target and possible ocean world. Astrobiology 20(2), 269-291 (2020). https://doi.org/10.1089/ast.2018.1999

17. Chiang, C.C., Kuan, Y.J., Chuang, Y.L.: ACA observations of Ceres' molecular exosphere. In: 16th AOGS Meeting, vol. PS16-A025 (2019)

18. Chuang, Y.L.: Submillimeter spectral observations of molecular exosphere of Ceres icy world. In: 16th AOGS Meeting, vol. PS16-A025 (2019)

19. Ciesla, F.J., Cuzzi, J.N.: The evolution of the water distribution in a viscous protoplanetary disk. Icarus 181(1), 178-204 (2006). https://doi.org/10.1016/j.icarus.2005.11.009, arXiv:astro-ph/0511372

20. Combe, J.P., McCord, T.B., Tosi, F., Ammannito, E., Carrozzo, F.G., De, S.anctis.M.C., Raponi, A., Byrne, S., Landis, M.E., Hughson, K.H.G., Raymond, C.A., Russell, C.T.: Detection of local $\mathrm{H}_{2} \mathrm{O}$ exposed at the surface of Ceres. Science 353(6303), aaf3010 (2016). https://doi.org/10.1126/science.aaf3010

21. Combe, J.P., Raponi, A., Tosi, F., De Sanctis, M.C., Carrozzo, F.G., Zambon, F., Ammannito, E., Hughson, K.H.G., Nathues, A., Hoffmann, M., Platz, T., Thangjam, G., Schorghofer, N., Schröder, S., Byrne, S., Landis, M.E., Ruesch, O., McCord, T.B., Johnson, K.E., Singh, S.M., Raymond, C.A., Russell, C.T.: Exposed $\mathrm{H}_{2} \mathrm{O}$-rich areas detected on Ceres with the dawn visible and infrared mapping spectrometer. Icarus 318, 22-41 (2019). https://doi.org/10.1016/j.icarus.2017.12.008

22. Connelly, J.N., Amelin, Y., Krot, A.N., Bizzarro, M.: Chronology of the solar system's oldest solids. ApJ 675(2), L121 (2008). https://doi.org/10.1086/533586

23. De Sanctis, M.C., Ammannito, E., Raponi, A., Marchi, S., McCord, T.B., McSween, H.Y., Capaccioni, F., Capria, M.T., Carrozzo, F.G., Ciarniello, M., Longobardo, A., Tosi, F., Fonte, S., Formisano, M., Frigeri, A., Giardino, M., Magni, G., Palomba, E., Turrini, D., Zambon, F., Combe, J.P., Feldman, W., Jaumann, R., McFadden, L.A., Pieters, C.M., Prettyman, T., Toplis, M., Raymond, C.A., Russell, C.T.: Ammoniated phyllosilicates with a likely outer Solar System origin on (1) Ceres. Nature 528(7581), 241-244 (2015). https://doi.org/10.1038/nature16172

24. De Sanctis, M.C., Raponi, A., Ammannito, E., Ciarniello, M., Toplis, M.J., McSween, H.Y., CastilloRogez, J.C., Ehlmann, B.L., Carrozzo, F.G., Marchi, S., Tosi, F., Zambon, F., Capaccioni, F., Capria, M.T., Fonte, S., Formisano, M., Frigeri, A., Giardino, M., Longobardo, A., Magni, G., Palomba, E., McFadden, L.A., Pieters, C.M., Jaumann, R., Schenk, P., Mugnuolo, R., Raymond, C.A., Russell, C.T.: Bright carbonate deposits as evidence of aqueous alteration on (1) Ceres. Nature 536(7614), 54-57 (2016). https://doi.org/10.1038/nature18290 
25. De Sanctis, M.C., Ammannito, E., McSween, H.Y., Raponi, A., Marchi, S., Capaccioni, F., Capria, M.T., Carrozzo, F.G., Ciarniello, M., Fonte, S., Formisano, M., Frigeri, A., Giardino, M., Longobardo, A., Magni, G., McFadden, L.A., Palomba, E., Pieters, C.M., Tosi, F., Zambon, F., Raymond, C.A., Russell, C.T.: Localized aliphatic organic material on the surface of Ceres. Science 355(6326), 719-722 (2017). https://doi.org/10.1126/science.aaj2305

26. De Sanctis, M.C., Ammannito, E., Raponi, A., Frigeri, A., Ferrari, M., Carrozzo, F.G., Ciarniello, M., Formisano, M., Rousseau, B., Tosi, F., Zambon, F., Raymond, C.A., Russell, C.T.: Fresh emplacement of hydrated sodium chloride on Ceres from ascending salty fluids. Nature Astronomy 4, 786-793 (2020). https://doi.org/10.1038/s41550-020-1138-8

27. DeMeo, FE., Carry, B.: The taxonomic distribution of asteroids from multi-filter all-sky photometric surveys. Icarus 226(1), 723-741 (2013). https://doi.org/10.1016/j.icarus.2013.06.027, arXiv:1307.2424

28. DeMeo, FE., Carry, B.: Solar System evolution from compositional mapping of the asteroid belt. Nature 505(7485), 629-634 (2014). https://doi.org/10.1038/nature12908, arXiv:1408.2787

29. Ermakov, A.I., Fu, R.R., Castillo-Rogez, J.C., Raymond, C.A., Park, R.S., Preusker, F., Russell, C.T., Smith, D.E., Zuber, M.T.: Constraints on ceres' internal structure and evolution from its shape and gravity measured by the dawn spacecraft. J. Geophys. Res. (Planets) 122(11), 2267-2293 (2017a). https://doi.org/10.1002/2017JE005302

30. Ermakov, A.I., Mazarico, E., Schröder, S.E., Carsenty, U., Schorghofer, N., Preusker, F., Raymond, C.A., Russell, C.T., Zuber, M.T.: Ceres's obliquity history and its implications for the permanently shadowed regions. Geophys. Res. Lett. 44(6), 2652-2661 (2017b). https://doi.org/10.1002/2016GL072250

31. Farinella, P., Vokrouhlický, D., Hartmann, W.K.: Meteorite delivery via yarkovsky orbital drift. Icarus 132(2), 378-387 (1998). https://doi.org/10.1006/icar.1997.5872

32. Fernandez, J.A., Ip, W.H.: Some dynamical aspects of the accretion of Uranus and Neptune: The exchange of orbital angular momentum with planetesimals. Icarus 58(1), 109-120 (1984). https://doi.org/10.1016/0019-1035(84)90101-5

33. Frigeri, A., De Sanctis, M.C., Ammannito, E., Tosi, F., Ciarniello, M., Zambon, F., Carrozzo, F.G., Raponi, A., McCord, T., Raymond, C.A., Russell, C.T.: The spectral parameter maps of Ceres from NASA/DAWN VIR data. Icarus 318, 14-21 (2019). https://doi.org/10.1016/j.icarus.2018.04.019

34. Fu, R.R., Ermakov, A.I., Marchi, S., Castillo-Rogez, J.C., Raymond, C.A., Hager, B.H., Zuber, M.T., King, S.D., Bland, M.T., Cristina De Sanctis, M., Preusker, F., Park, R.S., Russell, C.T.: The interior structure of Ceres as revealed by surface topography. Earth Planet. Sci. Lett. 476, 153-164 (2017). https://doi.org/10.1016/j.eps1.2017.07.053

35. Gladman, B.J., Migliorini, F., Morbidelli, A., Zappala, V., Michel, P., Cellino, A., Froeschle, C., Levison, H.F., Bailey, M., Duncan, M.: Dynamical lifetimes of objects injected into asteroid belt resonances. Science 277, 197-201 (1997). https://doi.org/10.1126/science.277.5323.197

36. Gomes, R., Levison, H.F., Tsiganis, K., Morbidelli, A.: Origin of the cataclysmic Late Heavy Bombardment period of the terrestrial planets. Nature 435(7041), 466-469 (2005). https://doi.org/10.1038/nature03676

37. Gradie, J., Tedesco, E.: Compositional structure of the asteroid belt. Science 216(4553), 1405-1407 (1982). https://doi.org/10.1126/science.216.4553.1405

38. Grant, R.: History of Physical Astronomy, from the earliest ages to the middle of the XIXth Century: Comprehending a detailed account of the establishment of the theory of gravitation by Newton, and its developement by his successors; with an exposition of the progress of research on all the other subjects of celestial physics. Bohn. https://books.google.de/books?id=1iUuAAAAcAAJ (1852)

39. Haddaji, A., Spry, J.A., Walter, N., Brucato, J.R., Rabbow, E., Rettberg, P., Kminek, G., Margheritis, D.B., McKenna-Lawlor, S., Fellous, JL., Yano, H., Cabezas, P., Sephton, M.: The International Planetary Protection Handbook (IPPH). In: 42nd COSPAR Scientific Assembly, vol. 42, pp. 3-14-18 (2018)

40. Hahn, J.M., Malhotra, R.: Orbital Evolution of Planets Embedded in a Planetesimal Disk. AJ 117(6), 3041-3053 (1999). https://doi.org/10.1086/300891, arXiv:astro-ph/9902370

41. Hargrove, K.D., Emery, J.P., Campins, H., Kelley, M.S.P.: Asteroid (90) Antiope: Another icy member of the Themis family? Icarus 254, 150-156 (2015). https://doi.org/10.1016/j.icarus.2015.03.008

42. Hartogh, P., Lis, D.C., Bockelée-Morvan, D., de Val-Borro, M., Biver, N., Küppers, M., Emprechtinger, M., Bergin, E.A., Crovisier, J., Rengel, M., Moreno, R., Szutowicz, S., Blake, G.A.: 
Ocean-like water in the Jupiter-family comet 103P/Hartley 2. Nature 478(7368), 218-220 (2011). https://doi.org/10.1038/nature10519

43. Hayne, P.O., Aharonson, O.: Thermal stability of ice on Ceres with rough topography. J. Geophys. Res. (Planets) 120(9), 1567-1584 (2015). https://doi.org/10.1002/2015JE004887

44. Hendrix, A.R., Hurford, T.A., Barge, L.M., Bland, M.T., Bowman, J.S., Brinckerhoff, W., Buratti, B.J., Cable, M.L., Castillo-Rogez, J., Collins, G.C., Diniega, S., German, C.R., Hayes, A.e.G., Hoehler, T., Hosseini, S., Howett, C.J.A., McEwen, A.S., Neish, C.D., Neveu, M., Nordheim, T.A., Patterson, G.W., Patthoff, D.A., Phillips, C., Rhoden, A., Schmidt, B.E., Singer, K.N., Soderblom, J.M., Vance, S.D.: The NASA roadmap to ocean worlds. Astrobiology 19(1), 1-27 (2019). https://doi.org/10.1089/ast.2018.1955

45. Hiesinger, H., Marchi, S., Schmedemann, N., Schenk, P., Pasckert, J.H., Neesemann, A., O’Brien, D.P., Kneissl, T., Ermakov, A.I., Fu, R.R., Bland, M.T., Nathues, A., Platz, T., Williams, D.A., Jaumann, R., Castillo-Rogez, J.C., Ruesch, O., Schmidt, B., Park, RS., Preusker, F., Buczkowski, D.L., Russell, C.T., Raymond, C.A.: Cratering on Ceres: Implications for its crust and evolution. Science 353(6303), aaf4758 (2016). https://doi.org/10.1126/science.aaf4759

46. Hillenbrand, L.A.: Disk-dispersal and planet-formation timescales. Physica Scripta Volume T 130, 014024 (2008). https://doi.org/10.1088/0031-8949/2008/T130/014024, arXiv:0805.0386

47. Hiroi, T., Zolensky, M.E., Pieters, C.M., Lipschutz, M.E.: Thermal metamorphism of the C, $\mathrm{G}, \mathrm{B}$, and $\mathrm{F}$ asteroids seen from the 0.7 micron, 3 micron and UV absorption strengths in comparison with carbonaceous chondrites. Meteorit. Planet. Sci. 31(3), 321-327 (1996). https://doi.org/10.1111/j.1945-5100.1996.tb02068.x

48. Hsieh, H.H., Jewitt, D.: A population of comets in the main asteroid belt. Science 312(5773), 561563 (2006). https://doi.org/10.1126/science.1125150

49. Huber, M.C.E., Schwehm, G.: Comet nucleus sample return - plans and capabilities. Space Sci. Rev. 56(1-2), 109-115 (1991). https://doi.org/10.1007/BF00178398

50. Jacobson, S.A., Morbidelli, A., Raymond, S.N., O’Brien, D.P., Walsh, K.J., Rubie, D.C.: Highly siderophile elements in Earth's mantle as a clock for the Moon-forming impact. Nature 508(7494), 84-87 (2014). https://doi.org/10.1038/nature13172, arXiv:1504.01421

51. Jolliff, B.L.: Introduction to special section: New Views of the Moon, Part 1, a series of papers related to the lunar science initiative New Views of the Moon enabled by combined remotely sensed and lunar sample data sets. J. Geophys. Res. 105(E2), 4173-4174 (2000). https://doi.org/10.1029/1999JE001237

52. Jones, T.D., Lebofsky, L.A., Lewis, J.S., Marley, M.S.: The composition and origin of the C, P, and D asteroids: Water as a tracer of thermal evolution in the outer belt. Icarus 88(1), 172-192 (1990). https://doi.org/10.1016/0019-1035(90)90184-B

53. Keil, K.: Thermal alteration of asteroids: evidence from meteorites. Planet. Space Sci. 48(10), 887903 (2000). https://doi.org/10.1016/S0032-0633(00)00054-4

54. King, S.D., Castillo-Rogez, J.C., Toplis, M.J., Bland, M.T., Raymond, C.A., Russell, C.T.: Ceres internal structure from geophysical constraints. Meteorit. Planet. Sci. 53(9), 1999-2007 (2018). https://doi.org/10.1111/maps.13063

55. Kokubo, E., Ida, S.: Orbital evolution of protoplanets embedded in a swarm of planetesimals. Icarus 114(2), 247-257 (1995). https://doi.org/10.1006/icar.1995.1059

56. Konopliv, A., Park, R., Vaughan, A., Bills, B., Asmar, S., Ermakov, A., Rambaux, N., Raymond, C., Castillo-Rogez, J., Russell, C., Smith, D., Zuber, M.: The ceres gravity field, spin pole, rotation period and orbit from the dawn radiometric tracking and optical data. Icarus 299, 411-429 (2018). https://doi.org/10.1016/j.icarus.2017.08.005

57. Krohn, K., Jaumann, R., Stephan, K., Otto, K.A., Schmedemann, N., Wagner, R.J., Matz, K.D., Tosi, F., Zambon, F., Gathen, I., Schulzeck, F., Schröder, S.E., Buczkowski, D.L., Hiesinger, H., McSween, H.Y., Pieters, C.M., Preusker, F., Roatsch, T., Raymond, C.A., Russell, C.T., Williams, D.A.: Cryogenic flow features on Ceres: Implications for crater-related cryovolcanism. Geophys. Res. Lett. 43(23), 11,994-12,003 (2016). https://doi.org/10.1002/2016GL070370

58. Kruijer, T.S., Burkhardt, C., Budde, G., Kleine, T.: Age of Jupiter inferred from the distinct genetics and formation times of meteorites. Proceedings of the National Academy of Science 114(26), 67126716 (2017). https://doi.org/10.1073/pnas.1704461114

59. Küppers, M., O’Rourke, L., Bockelée-Morvan, D., Zakharov, V., Lee, S., von Allmen, P., Carry, B., Teyssier, D., Marston, A., Müller, T., Crovisier, J., Barucci, M.A., Moreno, R.: Localized sources of water vapour on the dwarf planet (1)Ceres. Nature 505(7484), 525-527 (2014). https://doi.org/10.1038/nature12918 
60. Landis, M.E., Byrne, S., Schörghofer, N., Schmidt, B.E., Hayne, P.O., Castillo-Rogez, J., Sykes, M.V., Combe, J.P., Ermakov, A.I., Prettyman, T.H., Raymond, C.A., Russell, C.T.: Conditions for sublimating water ice to supply ceres' exosphere. J. Geophys. Res. (Planets) 122(10), 1984-1995 (2017). https://doi.org/10.1002/2017JE005335

61. Landis, M.E., Byrne, S., Combe, J.P., Marchi, S., Castillo-Rogez, J., Sizemore, H.G., Schörghofer, N., Prettyman, T.H., Hayne, P.O., Raymond, C.A., Russell, C.T.: Water vapor contribution to ceres' exosphere from observed surface ice and postulated Ice-Exposing impacts. J. Geophys. Res. (Planets) 124(1), 61-75 (2019). https://doi.org/10.1029/2018JE005780

62. Lecar, M., Podolak, M., Sasselov, D., Chiang, E.: On the Location of the Snow Line in a Protoplanetary Disk. ApJ 640(2), 1115-1118 (2006). https://doi.org/10.1086/500287, arXiv:astro-ph/0602217

63. Levison, H.F., Bottke, W.F., Gounelle, M., Morbidelli, A., Nesvorný, D., Tsiganis, K.: Contamination of the asteroid belt by primordial trans-Neptunian objects. Nature 460(7253), 364-366 (2009). https://doi.org/10.1038/nature08094

64. Levison, H.F., Morbidelli, A., Tsiganis, K., Nesvorný, D., Gomes, R.: Late Orbital Instabilities in the Outer Planets Induced by Interaction with a Self-gravitating Planetesimal Disk. AJ 142(5), 152 (2011). https://doi.org/10.1088/0004-6256/142/5/152

65. Magnani, P., Re, E., Fumagalli, A., Senese, S., Ori, G., Gily, A., Baglioni, P.: Testing of Exomars Em Drill Tool in Mars Analogous Materials Proceedings Advanced Space Technologies for Robotics and Automation (ASTRA) Noordwijk. European Space Agency, The Netherlands (2011)

66. Marchi, S., Barbieri, C., Küppers, M., Marzari, F., Davidsson, B., Keller, HU., Besse, S., Lamy, P., Mottola, S., Massironi, M., Cremonese, G.: The cratering history of asteroid (2867) Steins. Planet. Space Sci. 58(9), 1116-1123 (2010). https://doi.org/10.1016/j.pss.2010.03.017, arXiv:1003.5655

67. Marchi, S., Raponi, A., Prettyman, T.H., De Sanctis, M.C., Castillo-Rogez, J., Raymond, C.A., Ammannito, E., Bowling, T., Ciarniello, M., Kaplan, H., Palomba, E., Russell, C.T., Vinogradoff, V., Yamashita, N.: An aqueously altered carbon-rich Ceres. Nature Astronomy 3, 140-145 (2019). https://doi.org/10.1038/s41550-018-0656-0

68. McKinnon, W.B.: Where Did Ceres Accrete - In Situ in the Asteroid Belt, Among the Giant Planets, Or in the Primordial Transneptunian Belt? In: AAS/Division for Planetary Sciences Meeting Abstracts \#44, AAS/Division for Planetary Sciences Meeting Abstracts, p. 111.14 (2012)

69. McSween, H.Y., Emery, J.P., Rivkin, A.S., Toplis, M.J., Castillo-Rogez, J.C., Prettyman, T.H., De, S.anctis.M.C., Pieters, C.M., Raymond, C.A., Russell, C.T.: Carbonaceous chondrites as analogs for the composition and alteration of Ceres. Meteorit. Planet. Sci. 53(9), 1793-1804 (2018). https://doi.org/10.1111/maps.12947

70. Morbidelli, A., Levison, H.F., Tsiganis, K., Gomes, R.: Chaotic capture of Jupiter's Trojan asteroids in the early Solar System. Nature 435(7041), 462-465 (2005). https://doi.org/10.1038/nature03540

71. Morbidelli, A., Bitsch, B., Crida, A., Gounelle, M., Guillot, T., Jacobson, S., Johansen, A., Lambrechts, M., Lega, E.: Fossilized condensation lines in the Solar System protoplanetary disk. Icarus 267, 368-376 (2016). https://doi.org/10.1016/j.icarus.2015.11.027, arXiv:1511.06556

72. Nanne, J.A.M., Nimmo, F., Cuzzi, J.N., Kleine, T.: Origin of the non-carbonaceouscarbonaceous meteorite dichotomy. Earth Planet. Sci. Lett. 511, 44-54 (2019). https://doi.org/10.1016/j.eps1.2019.01.027

73. Nathues, A., Platz, T., Thangjam, G., Hoffmann, M., Mengel, K., Cloutis, E.A., Le Corre, L., Reddy, V., Kallisch, J., Crown, D.A.: Evolution of occator crater on (1) ceres. AJ 153(3), 112 (2017). https://doi.org/10.3847/1538-3881/153/3/112

74. Nathues, A., Platz, T., Thangjam, G., Hoffmann, M., Scully, J.E.C., Stein, N., Ruesch, O., Mengel, K.: Occator crater in color at highest spatial resolution. Icarus 320, 24-38 (2019). https://doi.org/10.1016/j.icarus.2017.12.021

75. Neesemann, A., van Gasselt, S., Schmedemann, N., Marchi, S., Walter, S.H.G., Preusker, F., Michael, G.G., Kneissl, T., Hiesinger, H., Jaumann, R., Roatsch, T., Raymond, C.A., Russell, C.T.: The various ages of Occator crater, Ceres: Results of a comprehensive synthesis approach. Icarus 320, 60-82 (2019). https://doi.org/10.1016/j.icarus.2018.09.006

76. O’Brien, D.P., Izidoro, A., Jacobson, S.A., Raymond, S.N., Rubie, D.C.: The Delivery of Water During Terrestrial Planet Formation. Space Sci. Rev. 214(1), 47 (2018). https://doi.org/10.1007/s11214-018-0475-8, arXiv:1801.05456

77. Okada, T., Iwata, T., Matsumoto, J., Chujo, T., Kebukawa, Y., Ito, M., Aoki, J., Kawai, Y., Yokota, S., Saito, S., Terada, K., Toyoda, M., Yabuta, H., Yurimoto, H., Matsuura, S., Tsumura, K., Yonetoku, D., Mihara, T., Matsuoka, A., Nomura, R., Yano, H., Hirai, T., Nakamura, R., Ulamec, S., Jaumann, R., Bibring, J.P., Grand, N., Szopa, C., Palomba, E., Helbert, J., Herique, A., Kumamoto, A., Grott, 
M., Auster, H.U., Klingelhoefer, G., Yoshida, F., Yoshikawa, M., Matsushita, M., Saiki, T., Kato, H., Mori, O., Kawaguchi, J.: OKEANOS - a Solar Power Sail Mission to a Jupiter Trojan Asteroid and Its Updated Science Mission Proposal. In: Lunar and Planetary Science Conference, Lunar and Planetary Science Conference, p. 1305 (2019)

78. Owen, T., Bar-Nun, A.: Comets, impacts, and atmospheres. In: Deep Earth and Planetary Volatiles, p. 35 (1994)

79. Platz, T., Nathues, A., Schorghofer, N., Preusker, F., Mazarico, E., Schröder, S.E., Byrne, S., Kneissl, T., Schmedemann, N., Combe, J.P., Schäfer, M., Thangjam, G.S., Hoffmann, M., GutierrezMarques, P., Landis, M.E., Dietrich, W., Ripken, J., Matz, K.D., Russell, C.T.: Surface waterice deposits in the northern shadowed regions of Ceres. Nature Astronomy 1, 0007 (2016). https://doi.org/10.1038/s41550-016-0007

80. Poole, G.M., Rehkämper, M., Coles, B.J., Goldberg, T., Smith, C.L.: Nucleosynthetic molybdenum isotope anomalies in iron meteorites - new evidence for thermal processing of solar nebula material. Earth Planet. Sci. Lett. 473, 215-226 (2017). https://doi.org/10.1016/j.epsl.2017.05.001

81. Prettyman, T.H., Yamashita, N., Toplis, M.J., McSween, H.Y., Schörghofer, N., Marchi, S., Feldman, W.C., Castillo-Rogez, J., Forni, O., Lawrence, D.J., Ammannito, E., Ehlmann, B.L., Sizemore, H.G., Joy, S.P., Polanskey, C.A., Rayman, M.D., Raymond, C.A., Russell, C.T.: Extensive water ice within Ceres' aqueously altered regolith: Evidence from nuclear spectroscopy. Science 355(6320), 55-59 (2017). https://doi.org/10.1126/science.aah6765

82. Prettyman, T.H., Yamashita, N., Ammannito, E., Castillo-Rogez, J.C., Ehlmann, B.L., McSween, H.Y., Marchi, S., Pieters, C.M., Schorghofer, N., Toplis, M.J., Russell, C.T., Raymond, C.A.: Carbon on Ceres: Implications for Origins and Interior Evolution. In: Lunar and Planetary Science Conference, Lunar and Planetary Science Conference, p. 1151 (2018)

83. Qin, L., Nittler, L.R., Alexander, C.M.O.D., Wang, J., Stadermann, F.J., Carlson, R.W.: Extreme ${ }^{54} \mathrm{Cr}$-rich nano-oxides in the CI chondrite Orgueil - Implication for a late supernova injection into the solar system. Geochim. Cosmochim. Acta 75(2), 629-644 (2011). https://doi.org/10.1016/j.gca.2010.10.017, arXiv:1101.4949

84. Quick, L.C., Buczkowski, D.L., Ruesch, O., Scully, J.E.C., Castillo-Rogez, J., Raymond, C.A., Schenk, P.M., Sizemore, H.G., Sykes, M.V.: A possible brine reservoir beneath occator crater: Thermal and compositional evolution and formation of the cerealia dome and vinalia faculae. Icarus $\mathbf{3 2 0}$, 119-135 (2019). https://doi.org/10.1016/j.icarus.2018.07.016

85. Raponi, A., De Sanctis, M.C., Frigeri, A., Ammannito, E., Ciarniello, M., Formisano, M., Combe, J.P., Magni, G., Tosi, F., Carrozzo, F.G., Fonte, S., Giardino, M., Joy, SP., Polanskey, C.A., Rayman, M.D., Capaccioni, F., Capria, M.T., Longobardo, A., Palomba, E., Zambon, F., Raymond, C.A., Russell, C.T.: Variations in the amount of water ice on Ceres' surface suggest a seasonal water cycle. Sci. Adv. 4(3), eaao3757 (2018). https://doi.org/10.1126/sciadv.aao3757

86. Raponi, A., De Sanctis, M.C., Carrozzo, F.G., Ciarniello, M., Castillo-Rogez, J.C., Ammannito, E., Frigeri, A., Longobardo, A., Palomba, E., Tosi, F., Zambon, F., Raymond, C.A., Russell, C.T.: Mineralogy of Occator crater on Ceres and insight into its evolution from the properties of carbonates, phyllosilicates, and chlorides. Icarus 320, 83-96 (2019). https://doi.org/10.1016/j.icarus.2018.02.001

87. Raymond, C.A., Ermakov, A.I., Castillo-Rogez, J.C., Marchi, S., Johnson, B.C., Hesse, M.A., Scully, J.E.C., Buczkowski, D.L., Sizemore, H.G., Schenk, P.M., Nathues, A., Park, R.S., Prettyman, T.H., Quick, L.C., Keane, J.T., Rayman, M.D., Russell, C.T.: Impact-driven mobilization of deep crustal brines on dwarf planet Ceres. Nat. Astron. 4, 741-747 (2020). https://doi.org/10.1038/s41550-020-1168-2

88. Raymond, SN., Izidoro, A.: Origin of water in the inner Solar System: Planetesimals scattered inward during Jupiter and Saturn's rapid gas accretion. Icarus 297, 134-148 (2017). https://doi.org/10.1016/j.icarus.2017.06.030, arXiv:1707.01234

89. Rivkin, A.S., Emery, J.P.: Detection of ice and organics on an asteroidal surface. Nature 464(7293), 1322-1323 (2010). https://doi.org/10.1038/nature09028

90. Rivkin, A.S., Howell, E.S., Vilas, F., Lebofsky, L.A.: Hydrated Minerals on Asteroids: The Astronomical Record, pp 235-253 (2002)

91. Rivkin, A.S., Thomas, C.A., Trilling, D.E., Mt, E.nga., Grier, J.A.: Ordinary chondritelike colors in small Koronis family members. Icarus 211(2), 1294-1297 (2011). https://doi.org/10.1016/j.icarus.2010.11.033

92. Roatsch, T., Kersten, E., Matz, K.D., Preusker, F., Scholten, F., Jaumann, R., Raymond, C., Russell, C.: High-resolution ceres low altitude mapping orbit atlas derived from dawn framing camera images. Planet. Space Sci. 140, 74-79 (2017). https://doi.org/10.1016/j.pss.2017.04.008 
93. Rousselot, P., Opitom, C., Jehin, E., Hutsemékers, D., Manfroid, J., Villarreal, M.N., Li, J.Y., Castillo-Rogez, J., Russell, C.T., Vernazza, P., Marsset, M., Roth, L., Dumas, C., Yang, B., Prettyman, T.H., Mousis, O.: Search for water outgassing of (1) Ceres near perihelion. A\&A 628, A22 (2019). https://doi.org/10.1051/0004-6361/201935738

94. Ruesch, O., Platz, T., Schenk, P., McFadden, L.A., Castillo-Rogez, J.C., Quick, L.C., Byrne, S., Preusker, F., O’Brien, D.P., Schmedemann, N., Williams, D.A., Li, J.Y., Bland, M.T., Hiesinger, H., Kneissl, T., Neesemann, A., Schaefer, M., Pasckert, J.H., Schmidt, B.E., Buczkowski, D.L., Sykes, M.V., Nathues, A., Roatsch, T., Hoffmann, M., Raymond, C.A., Russell, C.T.: Cryovolcanism on Ceres. Science 353(6303), aaf4286 (2016). https://doi.org/10.1126/science.aaf4286

95. Ruesch, O., Genova, A., Neumann, W., Quick, L.C., Castillo-Rogez, J.C., Raymond, C.A., Russell, C.T., Zuber, M.T.: Slurry extrusion on Ceres from a convective mud-bearing mantle. Nat. Geosci. 12(7), 505-509 (2019). https://doi.org/10.1038/s41561-019-0378-7

96. Russell, C.T., Raymond, C.A., Ammannito, E., Buczkowski, D.L., De Sanctis, M.C., Hiesinger, H., Jaumann, R., Konopliv, A.S., McSween, H.Y., Nathues, A., Park, R.S., Pieters, C.M., Prettyman, T.H., McCord, T.B., McFadden, L.A., Mottola, S., Zuber, M.T., Joy, S.P., Polanskey, C., Rayman, M.D., Castillo-Rogez, J.C., Chi, P.J., Combe, J.P., Ermakov, A., Fu, R.R., Hoffmann, M., Jia, Y.D., King, S.D., Lawrence, D.J., Li, J.Y., Marchi, S., Preusker, F., Roatsch, T., Ruesch, O., Schenk, P., Villarreal, M.N., Yamashita, N.: Dawn arrives at Ceres: Exploration of a small, volatile-rich world. Science 353(6303), 1008-1010 (2016). https://doi.org/10.1126/science.aaf4219

97. Sasselov, D.D., Lecar, M.: On the Snow Line in Dusty Protoplanetary Disks. ApJ 528(2), 995-998 (2000). https://doi.org/10.1086/308209, arXiv:astro-ph/9911390

98. Schmidt, B.E., Hughson, K.H.G., Chilton, H.T., Scully, J.E.C., Platz, T., Nathues, A., Sizemore, H., Bland, M.T., Byrne, S., Marchi, S., O’Brien, D.P., Schorghofer, N., Hiesinger, H., Jaumann, R., Pasckert, J.H., Lawrence, J.D., Buzckowski, D., Castillo-Rogez, J.C., Sykes, M.V., Schenk, P.M., Desanctis, M.C., Mitri, G., Formisano, M., Li, J.Y., Reddy, V., Lecorre, L., Russell, C.T., Raymond, C.A.: Geomorphological evidence for ground ice on dwarf planet Ceres. Nat. Geosci. 10(5), 338-343 (2017). https://doi.org/10.1038/ngeo2936

99. Schorghofer, N.: The lifetime of ice on main belt asteroids. Astrophys. J. 682(1), 697 (2008)

100. Schorghofer, N.: Predictions of depth-to-ice on asteroids based on an asynchronous model of temperature, impact stirring, and ice loss. Icarus 276, 88-95 (2016). https://doi.org/10.1016/j.icarus.2016.04.037

101. Schorghofer, N., Mazarico, E., Platz, T., Preusker, F., Schröder, S.E., Raymond, C.A., Russell, C.T.: The permanently shadowed regions of dwarf planet Ceres. Geophys. Res. Lett. 43(13), 6783-6789 (2016). https://doi.org/10.1002/2016GL069368

102. Schorghofer, N., Byrne, S., Landis, M.E., Mazarico, E., Prettyman, T.H., Schmidt, B.E., Villarreal, M.N., Castillo-Rogez, J., Raymond, C.A., Russell, C.T.: The putative cerean exosphere. ApJ 850(1), 85 (2017). https://doi.org/10.3847/1538-4357/aa932f

103. Scott, E.R.D., Taylor, G.J., Newsom, H.E., Herbert, F.L., Zolensky, M., Kerridge, J.F.: Chemical, Thermal and Impact Processing of Asteroids. In: Binzel, R.P., Gehrels, T., Matthews, M.S. (eds.) Asteroids II, pp. 701-739 (1989)

104. Scott, E.R.D., Krot, A.N., Sanders, I.S.: Isotopic Dichotomy among Meteorites and Implications for the Evolution of the Protoplanetary Disk. In: Lunar and Planetary Science Conference, Lunar and Planetary Science Conference, p. 1713 (2018)

105. Scully, J.E., Bowling, T., Bu, C., Buczkowski, D.L., Longobardo, A., Nathues, A., Neesemann, A., Palomba, E., Quick, L.C., Raponi, A., et al.: Synthesis of the special issue: The formation and evolution of ceres' occator crater. Icarus 320, 213-225 (2019a)

106. Scully, J.E., Buczkowski, D.L., Raymond, C.A., Bowling, T., Williams, D.A., Neesemann, A., Schenk, P.M., Castillo-Rogez, J.C., Russell, C.T.: Ceres' occator crater and its faculae explored through geologic mapping. Icarus 320, 7-23 (2019b)

107. Sizemore, H.G., Platz, T., Schorghofer, N., Prettyman, T.H., De Sanctis, M.C., Crown, D.A., Schmedemann, N., Neesemann, A., Kneissl, T., Marchi, S., Schenk, P.M., Bland, M.T., Schmidt, B.E., Hughson, K.H.G., Tosi, F., Zambon, F., Mest, S.C., Yingst, R.A., Williams, D.A., Russell, C.T., Raymond, C.A.: Pitted terrains on (1) Ceres and implications for shallow subsurface volatile distribution. Geophys. Res. Lett. 44(13), 6570-6578 (2017). https://doi.org/10.1002/2017GL073970

108. Sori, M.M., Byrne, S., Bland, M.T., Bramson, A.M., Ermakov, A.I., Hamilton, C.W., Otto, K.A., Ruesch, O., Russell, C.T.: The vanishing cryovolcanoes of Ceres. Geophys. Res. Lett. 44(3), 12431250 (2017). https://doi.org/10.1002/2016GL072319 
109. Sori, M.M., Sizemore, H.G., Byrne, S., Bramson, A.M., Bland, M.T., Stein, N.T., Russell, C.T.: Cryovolcanic rates on Ceres revealed by topography. Nature Astronomy 2, 946-950 (2018). https://doi.org/10.1038/s41550-018-0574-1

110. Takir, D., Emery, J.P.: Outer Main Belt asteroids: Identification and distribution of four 3- $\mu \mathrm{m}$ spectral groups. Icarus 219(2), 641-654 (2012). https://doi.org/10.1016/j.icarus.2012.02.022

111. Teets, D., Whitehead, K.: The discovery of ceres: How gauss became famous. Math. Mag. 72(2), 83-93 (1999)

112. Thomas, E.C., Vu, T.H., Hodyss, R., Johnson, P.V., Choukroun, M.: Kinetic effect on the freezing of ammonium-sodium-carbonate-chloride brines and implications for the origin of Ceres' bright spots. Icarus 320, 150-158 (2019). https://doi.org/10.1016/j.icarus.2017.12.038

113. Tosi, F., Carrozzo, F.G., Raponi, A., De Sanctis, M.C., Thangjam, G., Zambon, F., Ciarniello, M., Nathues, A., Capria, M.T., Rognini, E., Ammannito, E., Hoffmann, M., Krohn, K., Longobardo, A., Palomba, E., Pieters, C.M., Stephan, K., Raymond, C.A., Russell, C.T.: Mineralogy and temperature of crater Haulani on Ceres. Meteorit. Planet. Sci. 53(9), 1902-1924 (2018). https://doi.org/10.1111/maps.13078

114. Tosi, F., Carrozzo, F.G., Zambon, F., Ciarniello, M., Frigeri, A., Combe, J.P., De Sanctis, M.C., Hoffmann, M., Longobardo, A., Nathues, A., Raponi, A., Thangjam, G., Ammannito, E., Krohn, K., McFadden, L.A., Palomba, E., Pieters, C.M., Stephan, K., Raymond, C.A., Russell, C.T., Team, D.awn.S.cience.: Mineralogical analysis of the Ac-H-6 Haulani quadrangle of the dwarf planet Ceres. Icarus 318, 170-187 (2019). https://doi.org/10.1016/j.icarus.2017.08.012

115. Tsiganis, K., Gomes, R., Morbidelli, A., Levison, H.F.: Origin of the orbital architecture of the giant planets of the Solar System. Nature 435(7041), 459-461 (2005). https://doi.org/10.1038/nature03539

116. Villarreal, M.N., Russell, C.T., Luhmann, J.G., Thompson, W.T., Prettyman, T.H., A'Hearn, M.F., Küppers, M., O'Rourke, L., Raymond, C.A.: The dependence of the cerean exosphere on solar energetic particle events. ApJ 838(1), L8 (2017). https://doi.org/10.3847/2041-8213/aa66cd

117. Vinogradoff, V., Poggiali, G., Raponi, A., Ciarniello, M., De Angelis, S., Ferrari, M., CastilloRogez, J.C., Brucato, J., De Sanctis, M.C.: Laboratory investigations coupled to vir/dawn observations to quantify the large concentrations of organic matter on ceres. Minerals 11(7) https://doi.org/10.3390/min11070719, https://www.mdpi.com/2075-163X/11/7/719 (2021)

118. Walsh, K.J., Morbidelli, A., Raymond, S.N., O’Brien, D.P., Mandell, A.M.: A low mass for Mars from Jupiter's early gas-driven migration. Nature 475(7355), 206-209 (2011). https://doi.org/10.1038/nature10201, arXiv:1201.5177

119. Warren, P.H.: Stable-isotopic anomalies and the accretionary assemblage of the Earth and Mars: A subordinate role for carbonaceous chondrites. Earth Planet. Sci. Lett. 311(1), 93-100 (2011). https://doi.org/10.1016/j.eps1.2011.08.047

120. Weissman, P.R.: Diversity of Comets: Formation Zones and Dynamical Paths. Space Sci. Rev. 90, 301-311 (1999). https://doi.org/10.1023/A:1005278905101

121. Williams, D.A., Buczkowski, D.L., Crown, D.A., Frigeri, A., Hughson, K., Kneissl, T., Krohn, K., Mest, S.C., Pasckert, J.H., Platz, T., Ruesch, O., Schulzeck, F., Scully, J.E.C., Sizemore, H.G., Nass, A., Jaumann, R., Raymond, C.A., Russell, C.T.: High-Resolution Global Geologic Map of Ceres from NASA Dawn Mission. In: Planetary Geologic Mappers Annual Meeting, vol. 2066, p. 7001 (2018)

122. Zacny, K.: Drilling and Caching Architecture for the Mars2020 Mission. In: AGU Fall Meeting Abstracts, vol. 2013, pp. P51G-1797 (2013)

123. Zambon, F., Raponi, A., Tosi, F., De Sanctis, M.C., McFadden, L.A., Carrozzo, F.G., Longobardo, A., Ciarniello, M., Krohn, K., Stephan, K., Palomba, E., Pieters, C.M., Ammannito, E., Russell, C.T., Raymond, C.A.: Spectral analysis of Ahuna Mons from Dawn mission's visible-infrared spectrometer. Geophys. Res. Lett. 44(1), 97-104 (2017). https://doi.org/10.1002/2016GL071303

124. Zambon, F., Carrozzo, F.G., Tosi, F., Ciarniello, M., Combe, J.P., Frigeri, A., De Sanctis, M.C., Thangjam, G., Nathues, A., Hoffmann, M., Longobardo, A., Stephan, K., Raponi, A., Ammannito, E., Krohn, K., McFadden, L.A., Palomba, E., Raymond, C.A., Russell, C.T., Team, D.awn.S.cience.: Mineralogical analysis of quadrangle Ac-H-10 Rongo on the dwarf planet Ceres. Icarus 318, 212 229 (2019). https://doi.org/10.1016/j.icarus.2017.09.021

125. Zhang, T., Zhang, W., Wang, K., Gao, S., Hou, L., Ji, J., Ding, X.: Drilling, sampling, and samplehandling system for China's asteroid exploration mission. Acta Astronaut. 137, 192-204 (2017). https://doi.org/10.1016/j.actaastro.2017.04.017 
126. Zhang, X., Huang, J., Wang, T., Huo, Z.: Zhenghe - a Mission to a Near-Earth Asteroid and a Main Belt Comet. In: Lunar and Planetary Science Conference, Lunar and Planetary Science Conference, p. 1045 (2019)

127. Zou, Y., Li, W., Ouyang, Z.: China's Deep-space Exploration to 2030. Chinese Journal of Space Science 34(5), 516-517 (2014)

Publisher's note Springer Nature remains neutral with regard to jurisdictional claims in published maps and institutional affiliations. 


\section{Affiliations}

Xian Shi ${ }^{1,2}$ (D) . Julie Castillo-Rogez ${ }^{3} \cdot \mathrm{Henry} \mathrm{Hsieh}^{4} \cdot \mathrm{Hejiu} \mathrm{Hui}^{5}$. Wing-Huen $\mathrm{Ip}^{6}$. Hanlun Lei ${ }^{7}$. Jian-Yang $\mathrm{Li}^{4}$. Federico Tosi ${ }^{8}$. Liyong Zhou ${ }^{7}$. Jessica Agarwal ${ }^{1,9}$. Antonella Barucci ${ }^{10}$. Pierre Beck ${ }^{11}$.

Adriano Campo Bagatin ${ }^{12}$. Fabrizio Capaccioni ${ }^{8}$. Andrew J. Coates ${ }^{13}$. Gabriele Cremonese $^{14}$. Rene Duffard ${ }^{15}$. Manuel Grande ${ }^{16}$. Ralf Jaumann ${ }^{17}$. Geraint H. Jones ${ }^{13}$. Esa Kallio ${ }^{18}$. Yangting Lin ${ }^{19}$. Olivier Mousis ${ }^{20}$. Andreas Nathues $^{1}$. Jürgen Oberst ${ }^{21} \cdot$ Holger Sierks $^{1}$. Stephan Ulamec ${ }^{22}$. Mingyuan Wang ${ }^{23}$. The GAUSS Team

1 Max Planck Institute for Solar System Research, Göttingen, Germany

2 Present address: Shanghai Astronomical Observatory, Shanghai, China

3 NASA Jet Propulsion Laboratory, La Cañada Flintridge CA, USA

4 Planetary Science Institute, Tucson, AZ, USA

5 School of Earth Sciences and Engineering, Nanjing University, Nanjing, China

6 Institute of Astronomy and Space Science, National Central University, Chung Li, Taiwan

7 School of Astronomy and Space Science, Nanjing University, Nanjing, China

8 Istituto Nazionale di AstroFisica - Istituto di Astrofisica e Planetologia Spaziali (INAF-IAPS), Rome, Italy

9 Institute for Geophysics and Extraterrestrial Physics, Technical University Braunschweig, Braunschweig, Germany

10 LESIA-Observatoire de Paris, Université PSL, CNRS, Université de Paris, Sorbonne Université, F-92195 Meudon Principal Cedex, France

11 CNRS Institut de Planétologie et d'Astrophysique, Univ. Grenoble Alpes, Grenoble, France

12 Universidad de Alicante, Departamento de Física, Ingeniería de Sistemas y Teoría de la Señal, Alicante, Spain

13 Mullard Space Science Laboratory, University College London, Surrey, UK

14 INAF - Osservatorio Astronomico di Padova, Padova, Italy

15 Instituto de Astrofísica de Andalucía (CSIC), Granada, Spain

16 University of Aberystwyth, Aberystwyth, UK

17 Institute of Geological Sciences, Free University of Berlin, Berlin, Germany

18 School of Electrical Engineering, Aalto University, Aalto, Finland

19 Institute of Geology and Geophysics, Chinese Academy of Sciences, Beijing, China

20 Aix Marseille Univ, CNRS, CNES, LAM, Marseille, France

21 DLR Institute of Planetary Research, Berlin, Germany

22 DLR Space Operations and Astronaut Training, Cologne, Germany

23 National Astronomical Observatory, Chinese Academy of Science, Beijing, China 